\title{
1 Recent Advances in Tree Root Mapping and Assessment using Non- \\ 2 Destructive Testing Methods: a Focus on Ground Penetrating Radar
}

$4 \quad$ Amir M. ALANI ${ }^{1}$ and Livia LANTINI ${ }^{1}$

$5{ }^{1}$ School of Computing and Engineering, University of West London (UWL), St Mary's

6 Road, Ealing, London W5 5RF, UK

7 e-mail: Amir.Alani@uwl.ac.uk (*Corresponding author); Livia.Lantini@uwl.ac.uk

9 Abstract

10 This paper provides an overview of the existing literature on the subject of assessment and monitoring of tree roots and their interaction with the soil. An overview of tree root systems architecture is given, and the main issues in terms of health and stability of trees, as well as the impact of trees on the built environment, are discussed. An overview of the main destructive and non-destructive testing (NDT) methods is therefore given. The paper also highlights the lack of available research based outputs in the field of tree roots and soil interaction, as well as of the interconnectivity of tree roots with one another. Additionally, the effectiveness of non-destructive methods is demonstrated, in particular ground penetrating radar, in mapping tree root configurations and their interconnectivity. Furthermore, the paper references recent developments in estimating tree root mass density and health.

Keywords: assessment of tree roots; destructive testing methods; non-destructive testing methods; ground penetrating radar (GPR); tree root interconnectivity; tree root mass density. 


\section{Introduction}

The earliest identified fossil tree, from over 385 million years ago, was found in the New York State, USA in 2007. Trees and plants have always been part of life on planet Earth. The impact of trees and their value to human life and the environment have been discussed in numerous publications for decades, even centuries, as suggested in https://www.savatree.com/whytrees.html. In more detail, the value of trees within the context of modern life could be considered under the following areas:

- Ecological and environmental

- Community and social

- Aesthetics

- Commercial and economic

Trees and forests are every society's asset and must be looked after and cherished. The contribution of trees and their importance to environmental sustainability are so vast that they can only be compared to the existence of icebergs and our oceans. Prevention from destruction of trees and plants by cutting them at alarming rate for materialistic reasons (i.e. creating wealth in different shapes and forms) are vital to the preservation of life, both for humans and animals, on the planet Earth.

Likewise, safeguarding and having planned health monitoring and assessment of the existing trees and plants are equally important. Within this context, the understanding of the health of tree roots and plants (i.e. growth, architecture and interaction with the soil and other tree roots) are of paramount importance.

Appropriately managing and caring for natural heritage is more important than ever today (Innes, 1993), and there is a growing awareness of the need to protect the environment. In particular, the preservation of veteran or ancient trees presents a series of conservation challenges that differ from standard arboricultural practices.

Among all the tree organs, roots are of vital importance because they have crucial functions in plants and ecosystems: they provide anchorage, supply soil-borne resources and modify soil properties. However, even if roots account for between $10 \%$ and $65 \%$ of 
a tree's total biomass, they typically lie below the soil surface, which in turn has limited our understanding of tree root system development and their interaction with the surrounding environment.

Various methods have been used to study the root systems of plants. Such investigations are usually carried out using destructive methods, such as excavation or uprooting. Although these techniques can provide direct measurements of the roots, they are onerous, time-consuming and above all destructive. The damage that these techniques inflict on trees leads to a reduction in the number of measurements which can be carried out in the future, making it impossible to assess the status of the roots during a given period. Also, root systems are often destroyed by these inspection methods, thus becoming susceptible to infections and diseases which can lead to the death of the tree.

The use of non-destructive techniques for root inspection and analysis has gained popularity in recent years, as this method can provide information about tree root architecture without harming the tree. It also enables long-term monitoring of tree root systems, as no disturbance is caused to their development by the application of these techniques.

In this framework, ground penetrating radar (GPR) is widely acknowledged to be a powerful geophysical non-destructive tool, useful in locating buried objects such as bedrocks, artefacts, utilities infrastructure and objects, voids and sub-surface water levels. Recently, several studies have been carried out about the use of GPR for root detection and mapping, as well as for the estimation of root biomass and diameter. This technique has shown great potential due to the reliability of the results and its ease of use. However, some research has led to contradictory results, due probably to difficulties in surveying a non-homogeneous medium such as the soil-root system. For this reason, gaining comprehensive knowledge about tree root systems is advisable in order to improve the use of GPR in this field and the understanding of achieved results.

Hence, this review aims to evaluate state of the art in tree root system investigation, from the beginning to the most recent achievements in the non-destructive techniques field. To this purpose, a brief introduction on tree root system architecture is presented, 
82 to broaden the understanding of root growth, development and structure, as well as the 83 root system's dependence on the environment and the characteristics of the soil. 84 Following this, the main concerns regarding roots are defined and discussed, divided 85 into health problems which could affect roots and the damage that roots can cause to 86 the environment. The principal techniques for tree root system investigation are listed 87 and examined, from the destructive methods to the non-destructive techniques. The main achievements and limitations of each method are thus discussed.

89 Finally, a comprehensive review of GPR applications to root detection and root index quantifications is carried out, in a section organised as follows:

- GPR operating principles and signal processing techniques are outlined;

- The current state of knowledge about GPR use in tree root systems investigations is reviewed;

- Limiting factors to root surveys using GPR are outlined;

- Future perspectives are discussed. 


\section{Tree root systems architecture}

98 Tree roots are responsible for water and mineral uptake, carbohydrate storage and hormonal signalling (Pallardy, 2008), as well as for providing support and anchorage in the ground (Coutts, 1983). Thus, the health of the root system, and as a consequence the health of the tree, is closely linked to the soil conditions (Gregory, 2006).

Tree roots are usually composed of complex structures, and they can be divided into two main groups:

- Woody roots: roots that have gone through secondary growth, resulting in a

Root architecture is quite complex and varies between and within plant species (Gregory, 2006). As far as rooting depth is concerned, it is influenced not only by the tree species but also by the type and conditions of the soil (Stone \& Kalisz, 1991): in fact, the downward penetration of roots can be impeded by soils that are poorly aerated or 
126 too dense, and by the presence of rock layers or by low soil temperatures. Stone and

127 Kalisz (1991) carried out an extensive study on tree roots, reviewing the existing 128 literature and performing on-site surveys on a wide variety of tree species, demonstrating that root extent is strictly related to site conditions. Indeed, evidence has been found that many species can reach considerable depths if not limited by soil characteristics. According to Jackson et al. (1996), there can be significant differences in rooting depths, depending on the features of the surrounding environment: rooting profiles are shallowest in boreal forests, temperate grasslands, and tundra, due not only to the convenient characteristics of soil moisture and aeration but also the presence of physical barriers to root vertical growth, such as permafrost in tundras and some boreal forests (Bonan, 1992). On the other hand, root distribution is deeper in deserts and xeric shrublands, as the lack of water and nutrients in the shallow subsurface, together with extreme soil surface temperatures, inhibits root development in the upper soil layers (Nobel, 2003) and forces them to grow deeper. Regardless, there is undoubtedly a tendency for tree roots to be concentrated in the surface soil (Wilson, 1964) (Wang, et al., 2006), as it is usually better aerated and moist, it contains a higher concentration of minerals than the deeper layers. Pallardy (2008) states that root density is often higher in the first $30 \mathrm{~cm}$ below the soil surface.

144 On the other hand, root spread seems to be less closely related to soil temperature and characteristics (Strong \& La Roi, 1983). The extent of root development seems to rely upon the tree species, but also upon the stand density (Stone \& Kalisz, 1991) and the

147 presence of competing species (Shainsky \& Radosevich, 1992). Many rules of thumb have been presented for estimating root spread, the most common of which is a relation between root extent and canopy diameter (Tubbs, 1977); however, Stone and Kalisz

150 (1991) reported many examples of a maximum lateral root extent of more than 30m from the trunk, and in some cases more than $50 \mathrm{~m}$. This seems to demonstrate that roots tend to explore the largest soil area possible, in order to exploit its resources and provide anchorage and stability. These estimates commonly assume that there are few significant physical impediments to root extent; moreover, not much is known about 
how different trees compete for water and mineral uptake when root systems come in contact with one another.

\section{Main issues}

\subsection{Health and stability of the tree}

Tree diseases are an integral part of natural ecosystems, as they regulate the development of forests (Hansen \& Goheen, 2000). The coexistence of plants and pathogens is therefore necessary for the survival of both. However, human activities have often altered the natural balance, breaking down the geographical barriers that had preserved the ecosystems and allowing the movement of wild species (Richardson, et al., 2001). As a consequence of the increase in the global trade of plants, alien pathogens and fungi have invaded entire regions (Santini, et al., 2012) (Liebhold, et al., 2012), sometimes with devastating consequences, as in the Dutch elm disease (Gibbs, 1978) and the chestnut blight (Anagnostakis, 1987) cases. Such diseases not only have severe ecological consequences, but they can also have economic repercussions (Aukema, et al., 2011).

Fungal infections are one of the main causes of root disease, as fungi are natural components of forests (Hansen \& Goheen, 2000). These typically contaminate trees which have already been weakened by other factors, such as other pests or climatic changes (Williams, et al., 1986), and they usually spread from the roots of dead or uprooted trees (Rishbeth, 1972). Fungi penetrate the bark and initiate decay in roots, inducing root rot and infecting coarse roots and the lower stems of trees (Figure 1). 


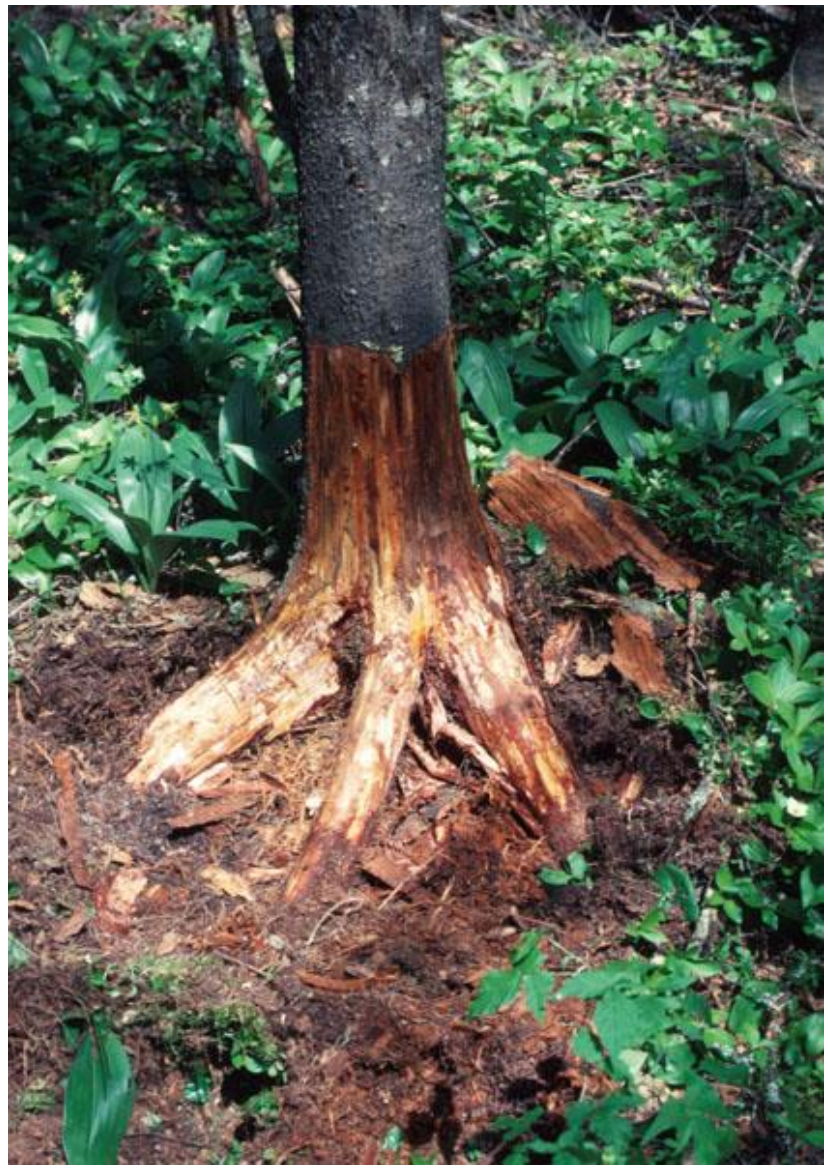

177 Figure 1: Roots and lower stem of a tree infected by Armillaria root rot fungi (Canadian Forest Service, 2015)

178 Plants can live for a long time even if sick, as they continue to collect water and nutrients 179 from healthy roots. Within this time, the infection can spread to other trees through 180 root contact (Hansen \& Goheen, 2000). Eventually, rotten roots will not be able to 181 provide anchorage and sustenance, and the contaminated tree will die either by windthrow or disease (Rishbeth, 1972).

183 The recognition of root diseases is difficult, as fungal infections do not show visible 184 symptoms. Manifestations of diseases can include the production of mushrooms around 185 the tree base, foliage discolouration and reduced growth (Williams, et al., 1986).

186 However, these symptoms can take several years to materialise if the tree is large or old, 187 and by the time the disease is recognised, it is often too late for any interventions. 


\subsubsection{Buildings}

191 Despite being an essential presence in urban and archaeological sites, trees can also 192 cause damage to structures and buildings. Damage can occur through direct contact with tree roots (Satriani, et al., 2010), as their growth can cause structures to uplift. This

194 is more likely to take place near the tree trunk, as the pressure exerted by roots 195 decreases rapidly with distance (MacLeod \& Cram, 1996). This usually occurs when trees are allocated an inadequate space: as the tree grows up, the roots start spreading and making their way underneath buildings (Day, 1991). The pressure that roots are capable of exerting is fairly weak and is further diminished by urban soil compaction (Roberts, et al., 2006). Moreover, modern building foundations are designed to withstand rootinducted movement.

201 Indirect damage is a more common cause of disturbance to structures, especially the shrinkage of expansive soils (Driscoll, 1983). Roots belonging to trees growing close to buildings tend to develop under the foundations, as the moisture content there tends to be higher than in the surrounding soil (Figure 2). The extraction of water by roots creates a reduction in soil volume, resulting in subsidence and cracks in the structures (Day, 1991).

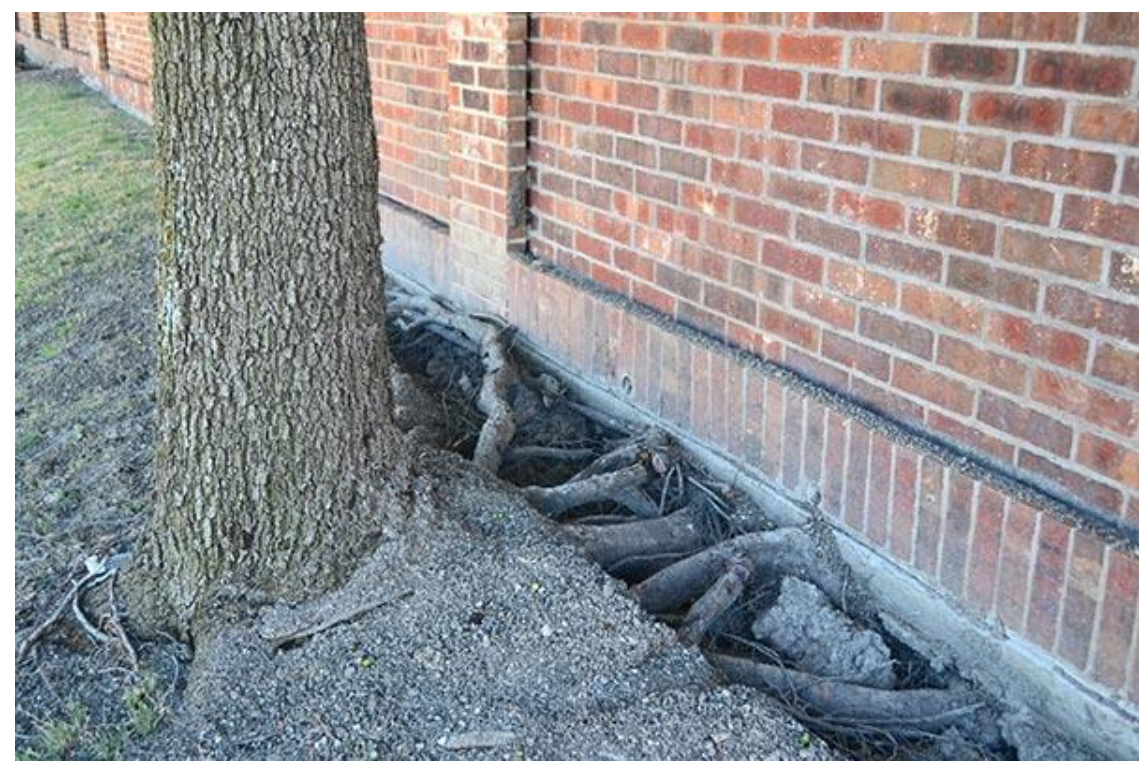


209 Cutler and Richardson (1981) and Biddle (2001) have reviewed several cases of damage

210 to buildings, producing an extensive analysis of how tree root interaction with the

211 surrounding environment can damage buildings. Regarding damage to ancient

212 structures, Caneva, Ceschin and De Marco (2006) have carried out a risk evaluation of

213 root-induced damage which archaeological sites are exposed to, while Caneva et al.

214 (2009) have surveyed the archaeological site of Villa Torlonia in Italy, investigating the 215 root expansion and evaluating the tendency of various species to harm ancient 216 monuments.

\subsubsection{Utilities}

218 Underground services, especially sewers, are frequently obstructed or damaged by the growth of roots. This damage usually occurs in old systems (Randrup, et al., 2001), as these were built with materials which could deteriorate with time, such as bricks or concrete. Moreover, roots are attracted by the presence of moisture around pipes, which are commonly cooler than the surrounding soil (Brennan, et al., 1997) and tend to grow around the pipe (Figure 3).

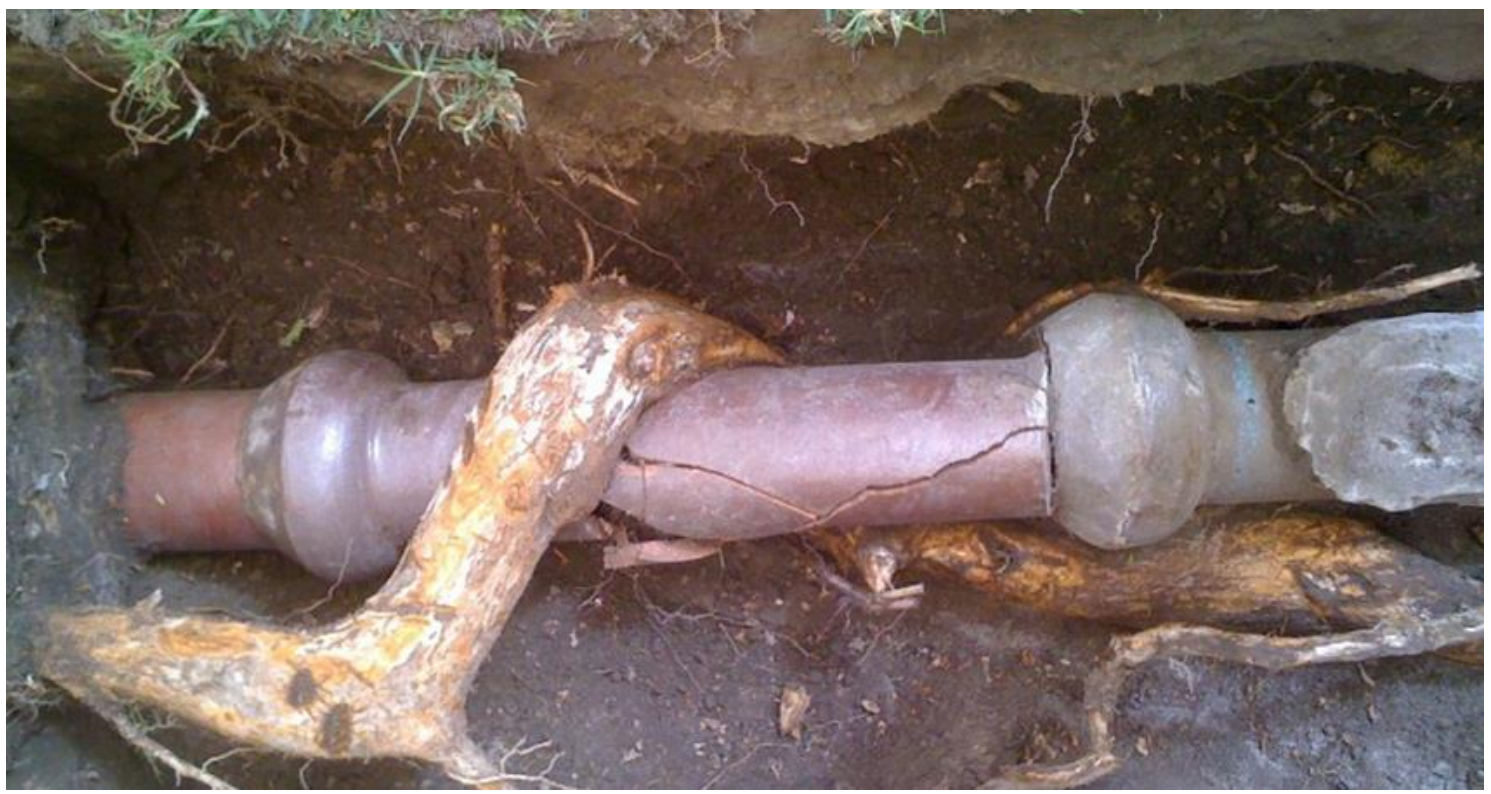


Modern sewers are made of plastic, iron or reinforced concrete, which are unlikely to be damaged by root growth pressure. Potential leakages due, for example, to a broken joint (Schrock, 1994) or poor construction (Sullivan, et al., 1977) (Brennan, et al., 1997) can lead to roots penetrating the pipe, and eventually blocking it.

231

232

\subsubsection{Roads and pavements}

Urban trees provide several environmental, social and economic benefits, but they can also cause extensive damage to road infrastructures. Root development can cause disruptions to road surfaces, such as cracking or uplifting (Francis, et al., 1996) (Figure 4). This damage can have serious consequences (Tosti, et al., 2018a), leading to additional pavement maintenance or repair and interventions on the tree (Mullaney, et al., 2015).

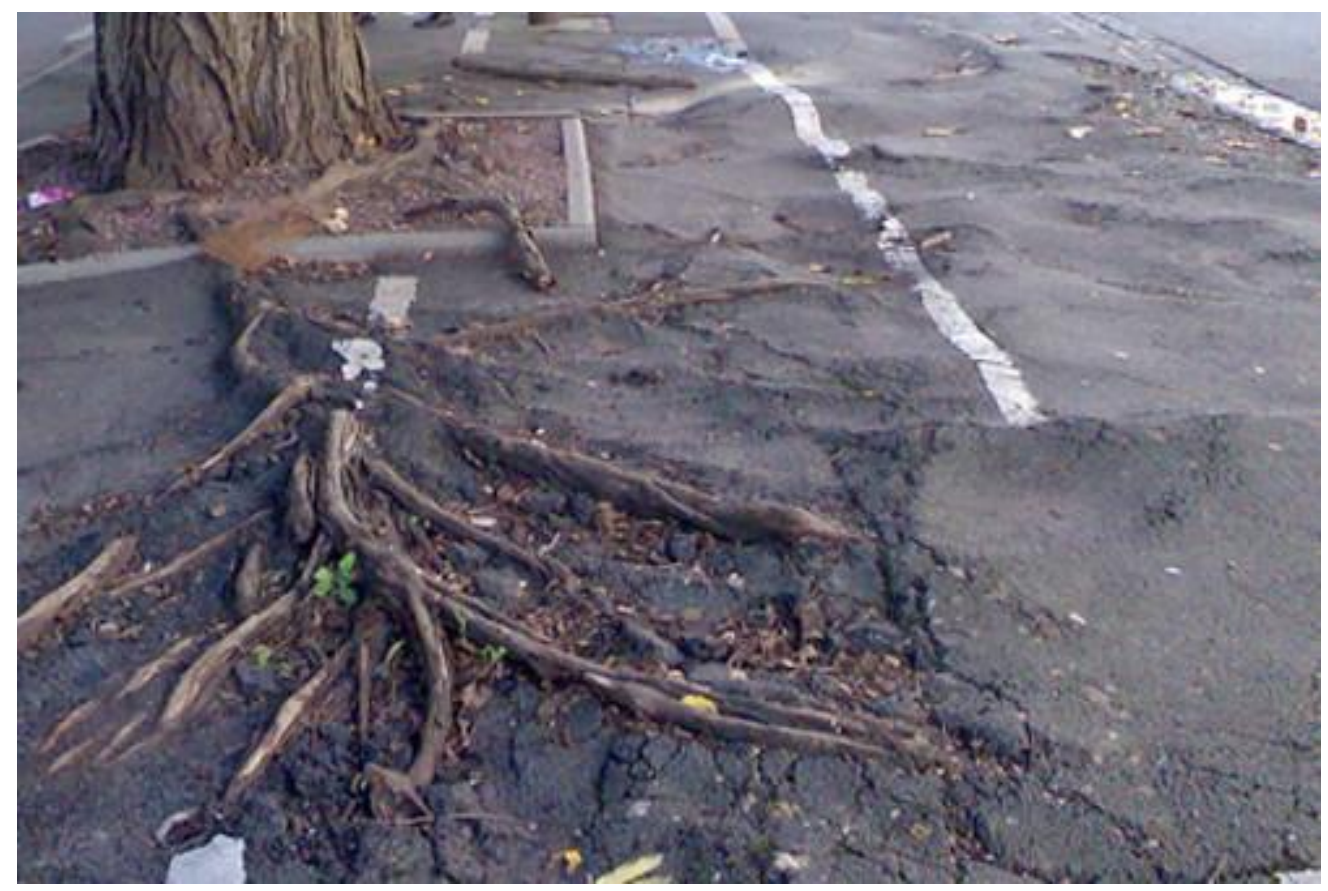

Figure 4: Damages to road pavement due to tree roots

One of the principal causes of conflict between roots and infrastructures seems to be the limited space provided for the development of trees (Barker, 1983) (Francis, et al., 1996). Tree size at maturity should be considered when choosing tree species to plant, as it will influence the necessary volume of soil (Trowbridge \& Bassuk, 2004). Such amounts of soil are not typical of urban environments, and trees are usually confined to 
245 tree lawns, which restrict not only the roots but also the branch and canopy 246 development (Pokorny, et al., 2003). Also, trunk flare and root buttresses are associated 247 with road infrastructure damages (Wagar \& Barker, 1983), and the tendency of species 248 to develop them should be considered when choosing which tree to plant (Costello \& 249 Jones, 2003). Finally, when large trees are planted in cities, there is a significant danger 250 of wind-throw, as tree roots are often cut during pavement repairs and therefore cannot 251 offer sufficient resistance to wind load (Pokorny, et al., 2003). Therefore, a selection of 252 species adequately matched to the site conditions is advisable (Costello \& Jones, 2003), 253 as this can lead to a significant reduction of hazards; however, McPherson and Peper 254 (2000) state that this resolution would reduce the benefits gained from larger trees.

255 Another factor which limits root development is soil compaction, as it decreases soil 256 aeration, restricts air and water movement, limits water-holding capacity and impedes root penetration (Boyer, 1995). This is a significant issue in urban areas, as it conflicts with road engineering specifications, which require a load-bearing base to support pavement loading (Grabosky, et al., 1998). The essential requirement is to increase soil compaction in order to reduce cavities and increase contact between the grains, thus giving the lithic structure a high frictional resistance. Moreover, this minimises deferred subsidence, providing greater functionality and security to the infrastructure. The resulting level of compaction produces unbearable conditions for root growing (Blunt, 2008) (Grabosky, et al., 2009) as it limits access to oxygen, water and nutrients (Loh, et al., 2003) (Lucke, et al., 2011) (Tracy, et al., 2011). Table 1 compares the prescriptions for bulk densities of soils based on the Proctor Compaction Test (ASTM D698/AASHTO T99) with the maximum level of compaction, which inhibits root penetration. 


\begin{tabular}{|c|c|c|c|c|c|c|}
\hline \multicolumn{7}{|c|}{ Bulk density of soils at $70-95 \%$ relative compaction } \\
\hline & & \multicolumn{2}{|c|}{ Landscape } & \multicolumn{2}{|c|}{ Paving } & \multirow{2}{*}{ Critical bulk density } \\
\hline & & $70 \%$ & $85 \%$ & $90 \%$ & $95 \%$ & \\
\hline \multirow{7}{*}{$\frac{\stackrel{ \pm}{2}}{\frac{0}{\overline{0}}}$} & Loamy sand (WG) & 1.52 & 1.85 & 1.96 & 2.07 & 1.75 \\
\hline & Sandy loam (WG) & 1.43 & 1.74 & 1.85 & 1.95 & 1.70 \\
\hline & Sandy loam (MG) & 1.35 & 1.64 & 1.74 & 1.83 & 1.70 \\
\hline & Sandy silty clay & 1.29 & 1.56 & 1.66 & 1.75 & 1.50 \\
\hline & Silt & 1.19 & 1.45 & 1.53 & 1.62 & 1.40 \\
\hline & Silty clay & 1.22 & 1.49 & 1.58 & 1.66 & 1.40 \\
\hline & Clay & 1.15 & 1.40 & 1.49 & 1.57 & 1.40 \\
\hline
\end{tabular}

Table 1: Information on the critical bulk density for soils of differing textures (ASTM D698/AASHTO T99). Critical bulk density is the level of compaction at which the roots are no longer able to penetrate the soil. Units are given as dry bulk density in grams per cubic centimetre (gm/cc). WG is with gravel; MG is minus gravel (Lindsey \& Barlow, 1994)

273 Such levels of compaction cause roots to develop at the interface between the pavement and soil, where nutrients and moisture are available (Kopinga, 1994)

275 (Randrup, et al., 2001) (Wagar \& Franklin, 1994). The favourable conditions that roots

276 find at the interface between the surface layer and the sub-base make them grow faster,

277 resulting in accelerated secondary thickening that can cause damage to the road surface

278 (Nicoll \& Armstrong, 1998).

279 Other issues that can interfere with root growth in urban environments and lead to road 280 infrastructure damage are waterlogging (Boyer, 1995) (Pokorny, et al., 2003) and severe water deficiency (Boyer, 1995) (Mullaney, et al., 2015). In the former case, soil saturation displaces air, making soil aeration more restrictive as depth increases and therefore forcing roots to grow within the soil surface; these conditions encourage the development of root pathogens. In the latter case, water deficit causes trees to slow down their leaf growth, resulting in a surplus of carbohydrates, which then become available for root growth. The immediate consequence, therefore, is that the root dimensions of water-stressed plants are higher than average. 


\section{Detection and Mapping of Tree Root Systems}

Locating tree roots and estimating their depth and spread is a significant challenge, and a necessary condition for several practices, ranging from tree health preservation to safety assessment in urban areas. There are several methods for studying roots available, which can be divided into destructive or non-destructive techniques.

\subsection{Destructive testing methods}

Destructive testing methods allow for the investigation of root systems at the time of sampling. Therefore, they are of limited value for investigating developmental processes. Moreover, these techniques are not only destructive to the root system itself and its immediate environment (Taylor, et al., 1991), but are also expensive, timeconsuming and laborious (Krainyukov \& Lyaksa, 2016). Given root system architecture variability, several replicated samples are needed to precisely assess root parameters, but this practice destroys the roots and exposes the tree to diseases and infections that can lead to its death (Smit, et al., 2013). However, these techniques are still widely used, as they provide reliable quantitative results.

The main destructive techniques are:

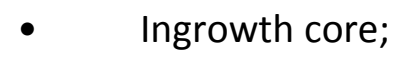

- $\quad$ Auger method;

- $\quad$ Monolith method.

\subsubsection{Ingrowth core}

Ingrowth cores are commonly used to quantify fine root production and to estimate the rate of growth during a given period (Smit, et al., 2013). They are also adopted to examine the effect of experimental manipulation on root growth (Majdi, et al., 2005). The operating principle of this technique is to replace a volume of soil (as it is) with the same volume of root-free soil, enclosed in a mesh bag, which is resampled after a determined period (Figure 5). This method is widely acknowledged to be straightforward and inexpensive, and it illustrates how long it takes for roots to develop in a particular soil. However, it can lead to misinterpretation, as the soil structure is altered when the 

growth rates. Moreover, since roots are damaged by the initial coring, their development into the root-free samples can be unnatural (Majdi, et al., 2005).

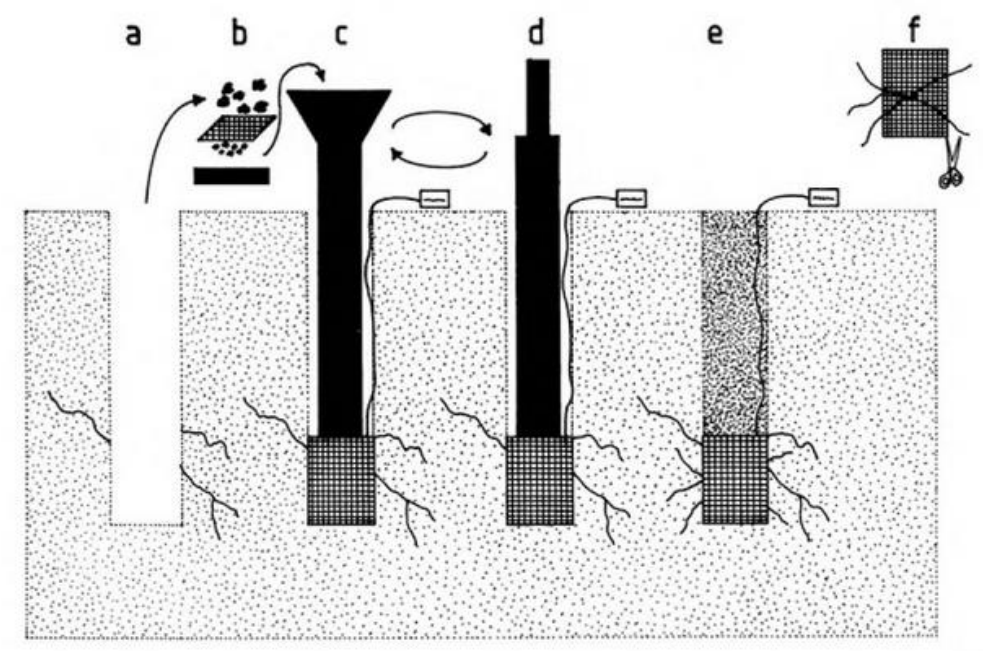

Figure 5: Procedure for installing the mesh bags for the root ingrowth core technique (Smit, et al., 2013). a) a core of soil is removed and b) the soil is sieved to remove the roots; c) a mesh bag is placed in the hole, which is filled with the sieved soil; d) the soil is packed to the original bulk density by means of a pestle; e) the mesh bag is left in place for a determined period of time, after which it is recovered and f) non-woody roots are trimmed.

\subsubsection{Auger method}

The auger method is the most convenient for investigating root density. It involves taking soil samples from the field, which are then washed to separate roots from the soil (Bohm, 2012) (Smit, et al., 2013). The soil core extraction can be made using either a hand-operated or a mechanical sampler, depending on the hardness of the investigated soil. The former is faster to use, being a cylindrical tube $15 \mathrm{~cm}$ long with an inside diameter of $7 \mathrm{~cm}$, equipped with a T-handle at the top that simplifies the penetration into the soil by rotation. However, if core samples need to be taken from hard soil or considerable depths, the auger is driven into the soil by a motorised dropping hammer, and then pulled back using a screw-jack (Smit, et al., 2013).

There exists uncertainty about the frequency of samples required in order to obtain reliable results (Bohm, 2012), however, increasing the number of samples will lower the uncertainty and improve the variability of data collected (Smit, et al., 2013). 


$$
\text { A }
$$

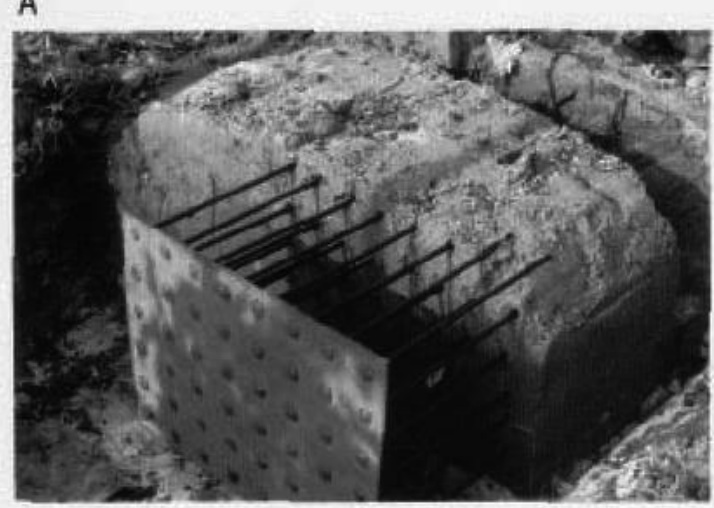

\subsubsection{Monolith method} is washed away (Boyer, 1995) (Figure 6).
B

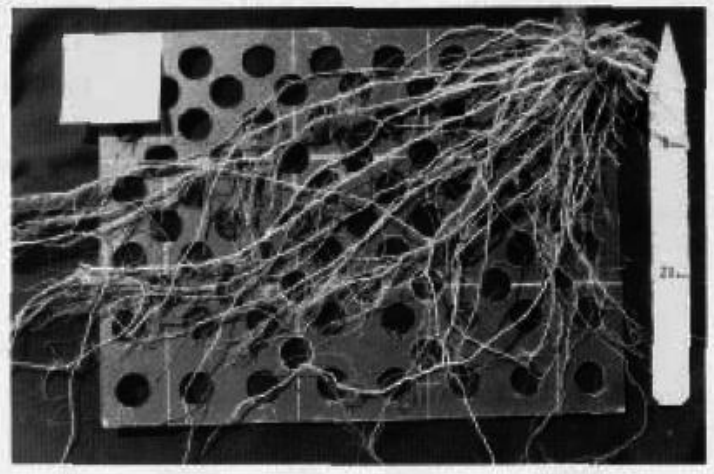

351

The monolith method requires large blocks of soil to be removed and washed out, in order to separate the roots from the soil (Boyer, 1995) (Bohm, 2012). Contrary to the auger method, which requires just the root volume to be quantified, in this technique roots are washed without displacing them from their original position (Weaver \& Voigt, 1950). This is possible thanks to the use of special boards covered with spikes, called pinboards, which are driven into the soil to preserve the root architecture while the soil

Figure 6: Metallic monolith pinboard used for excavating the soil-root samples (left) and roots after extraction and washing from the soil (right) (Leskovar, et al., 1994)

This technique provides useful information, as it is possible to have a general view of the root system architecture (Smit, et al., 2013). On the other hand, the collection of the samples requires great skill in order not to displace the roots, so the pinboards are usually of limited dimensions; additionally, the washing process can introduce biases, as significant losses of fine roots can occur (Smit, et al., 2013). Finally, this method is often non-repeatable, as the hole will be filled up with new soil that could lead the roots to develop differently, affecting the results of a second inspection (Schuurman \& Goedewaagen, 1965). 


\subsection{Non-destructive testing methods}

363 Non-destructive evaluations are acknowledged as being effective in investigating 364 different materials, without harming or damaging them (Buza \& Divos, 2016). 365 Furthermore, these techniques are easily repeatable, which means that long-term 366 investigation and monitoring of trees can be achieved (Buza \& Divos, 2016).

367 The main non-destructive techniques applied in root system investigations are:

- Rhizotrons and minirhizotrons;

- Pulling test;

- Electrical resistivity tomography (ERT);

- Acoustic detection;

- X-ray computed tomography (CP);

- Ground penetrating radar (GPR).

\subsubsection{Rhizotrons and minirhizotrons}

375 One of the first NDT methods for tree root system observations was to put glass plates

376 into the soil, so that it was possible to observe root development and growth against

377 them. This method has evolved into the modern rhizotron, namely an underground 378 chamber equipped with glass walls (Boyer, 1995).

379 This technique provides repeated and non-destructive access to soil and roots, allowing 380 for a better understanding of underground processes as they are in nature. Nevertheless, since such an instalment is impossible to set up for assessment of urban trees, minirhizotrons have become increasingly popular. These instruments consist of small plastic tubes (about $5 \mathrm{~cm}$ in diameter and 2 to $3 \mathrm{~m}$ long), which can be driven into the ground at different angles (Majdi, 1996). A fibre optic light and a camera are then lowered down the tube, in order to observe the roots' developmental process over time

386 (Boyer, 1995), sometimes in combination with dedicated image processing software 387 (Majdi, 1996) (Figure 7). 


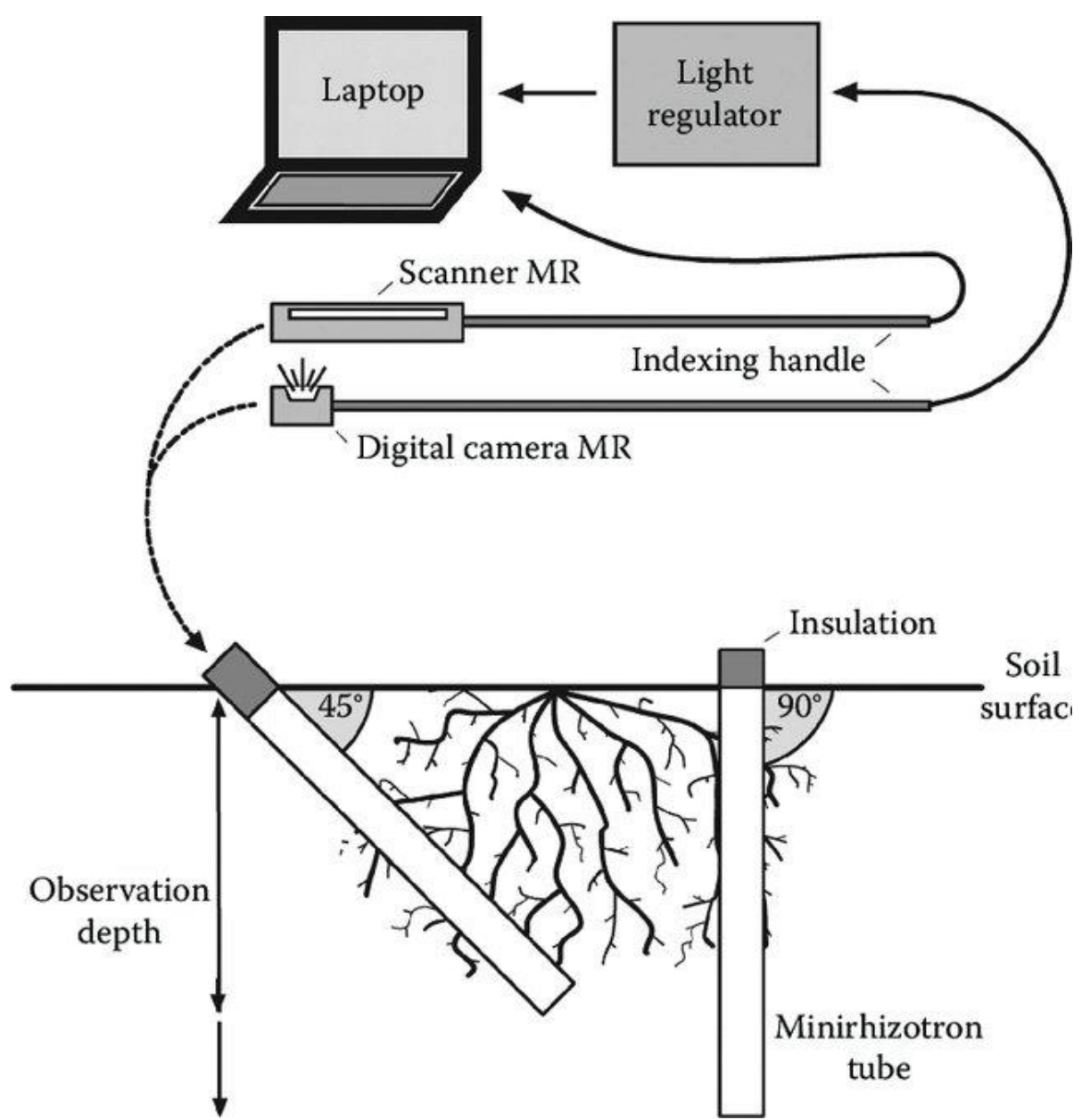

This method is commonly used for quantitative investigations on root length production, root length mortality, longevity, rooting density and root diameter, as well as to achieve qualitative information about root colour, branching and decomposition (Majdi, 1996).

The main limitations of this technique are linked to its installation in hard or stony soils (Majdi, 1996). Moreover, the viewing window is static, providing only a limited, 2-D visualisation that is unrepresentative of the architecture of a tree root system (Mooney, et al., 2012). Another limitation arises from the fact that rhizotrons are not totally noninvasive, as they may create an altered soil-root interface that could affect root growth (Amato, et al., 2009) (Neumann, et al., 2009). Finally, the effectiveness of minirhizotrons as opposed to other techniques, especially when used in the shallow subsurface, is still an object of discussion (Heeraman \& Juma, 1993). 
402

The pulling test is principally applied to test the root system anchorage to the soil. Its

404 primary application is the assessment of the reaction of the tree to a determined load, 405 especially the one caused by the wind (Buza \& Divos, 2016), in terms of the resulting bending of the stem and the inclination of the root plate (Fay, 2014).

407 During a pulling test, a load is applied to the subject tree by securing a cable to the tree 408

Divos, 2016) (Marchi, et al., 2018). In order to evaluate the risk of tree uprooting, an inclinometer is applied to the trunk close to the ground. Depending on the tree species and conditions, limits are placed on the possible inclination of the tree, in order to prevent damage to tree roots. Destructive pulling tests were conducted in several studies (Coutts, 1983) (Brudi \& Wassenaer, 2002) (Lundström, et al., 2007), which report root failure models and maximum inclination values for different tree species.
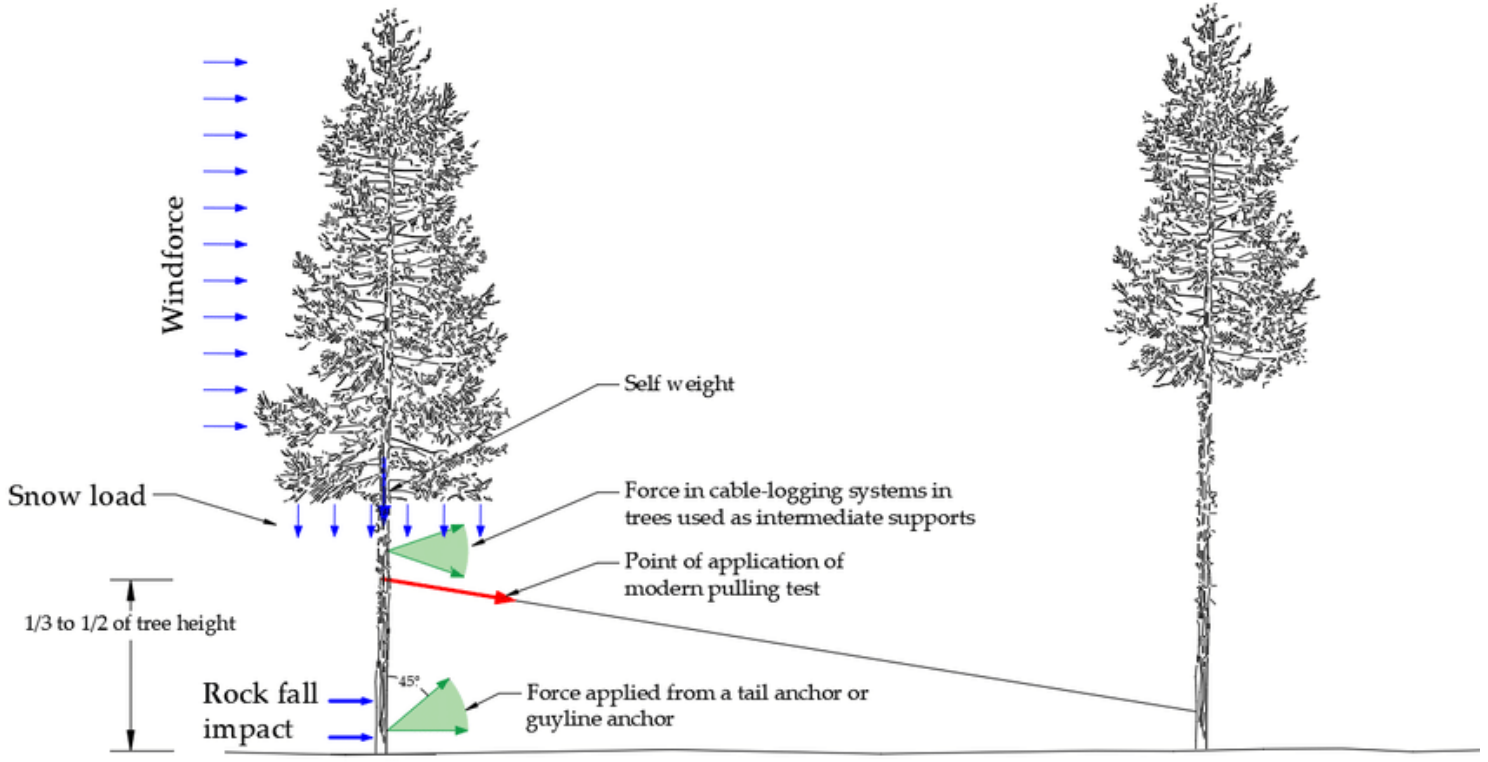

418 The primary output of a pulling test is a safety factor, which is given by the ratio between 419 the tree capacity and the calculated load (Buza \& Divos, 2016). According to field studies 420 (Fay, 2014), a tree is considered stable when its safety factor is greater than 1.5. 
421 The pulling test provides useful information on the stability of trees, evaluating their

422 resistance to external loads. It can be performed not only to assess the tree root plate

423 conditions, but also the status of the trunk in terms of maximum bending moment (Fay,

424 2014). However, the main limitation of this method is that it is not completely non-

425 invasive, as both the trunk and the roots can be damaged when the pulling force is 426 applied (Marchi, et al., 2018).

427 Other limitations to this methodology arise from the fact that the applied load cannot 428 represent the complex action of the wind, but can only cause a reaction in the tree which 429 can be compared to the one produced by the wind load (Fay, 2014). Moreover, the test 430 could be affected by factors such as the temperature conditions of both the soil and the 431 tree (Buza \& Divos, 2016). Finally, the pulling test cannot predict the moment or the 432 conditions under which the tree will fail (Fay, 2014), but can only assess the conditions 433 of the tree at the time of testing.

\subsubsection{Electrical resistivity tomography}

435 Electrical resistivity tomography (ERT) is a geophysical technique used for the calculation 436 of the subsurface distribution of soil electrical resistivity (Zenone, et al., 2008). Electrical 437 resistivity $(\rho)$ is defined as the electrical resistance through a uniform body of unit length 438 and unit cross-sectional area and represents a measure of the ability of materials to limit 439 the transfer of electrical current. This method has been extensively used for the 440 characterisation of soil heterogeneity.

441 Soil resistivity is measured by applying electric currents through at least two conductors 442 (current electrodes) and measuring the resulting differences in electric potential 443 (voltage) on at least two separate conductors (potential electrodes). There are different 444 possible geometric configurations for electrodes. The potential electrodes could be 445 placed between the current electrodes (Wenner array, Figure 9) or consecutive to them 446 (dipole-dipole configuration). The investigation depth relies on the configuration choice, 447 and increases with the spacing between electrodes (Amato, et al., 2009). 


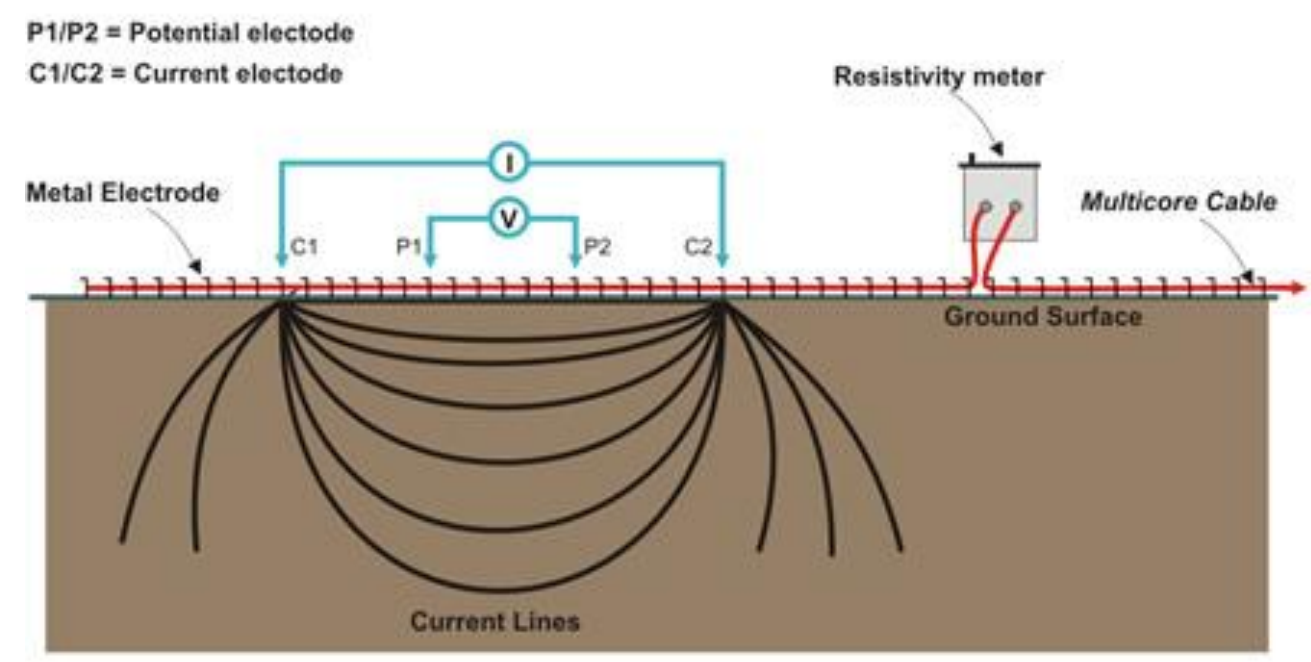

448

Figure 9: General ERT operating principles for a Wenner array configuration

450 The voltage distribution in space is a function of the different resistivity of soil volumes

451 (Kearey, et al., 2013).

452 Geophysical surveys performed using electrical exploration have qualitative purposes, 453 and are based on the contrast between the resistivity of different soil layers or the 454 heterogeneous materials within each layer. In heterogeneous media, the current flow 455 lines are deformed and tend to be concentrated in conductive volumes. Resistivities are 456 first calculated according to the theoretical flow-line distribution in isotropic media and 457 are called apparent resistivity values. These are attributed to soil coordinates 458 corresponding to the hypothesis of homogeneous current distribution and arranged in 459 a pseudosection. In order to obtain real resistivity values, correctly positioned in space 460 (true section), a procedure called inversion is applied. The investigated soil domain is 461 divided into elementary cells, and resistivity data are imaged by attributing values 462 corresponding to each elementary soil volume to a point corresponding to the 463 intersection of two lines conducted through the centres of the quadrupoles (Figure 10) 464 (Amato, et al., 2009). 
(a) Current electrodes

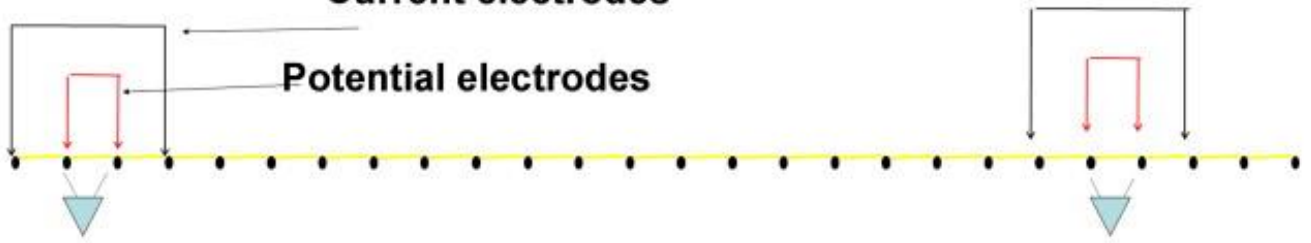

a

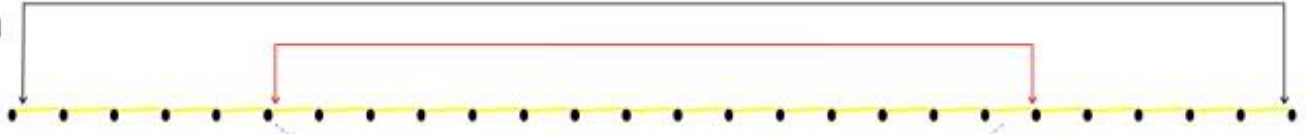

a

(b)

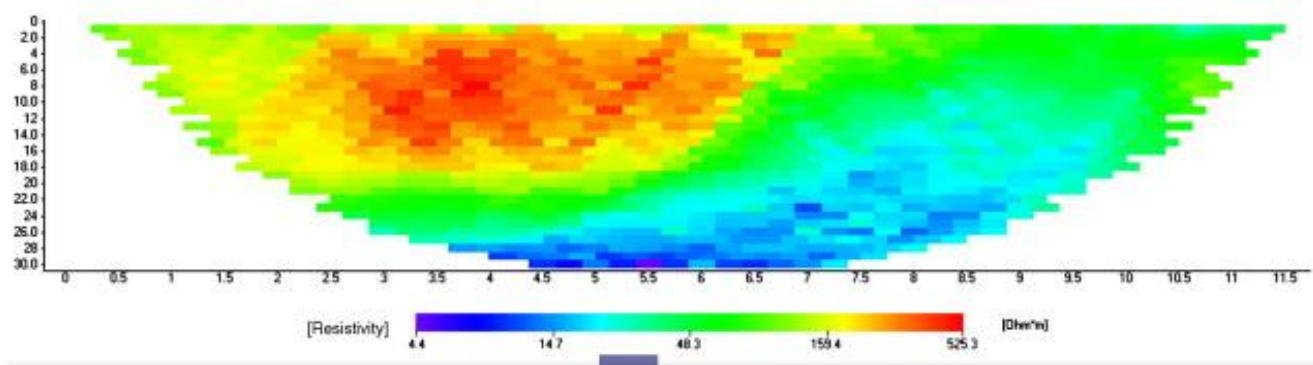

Data inversion

(c)

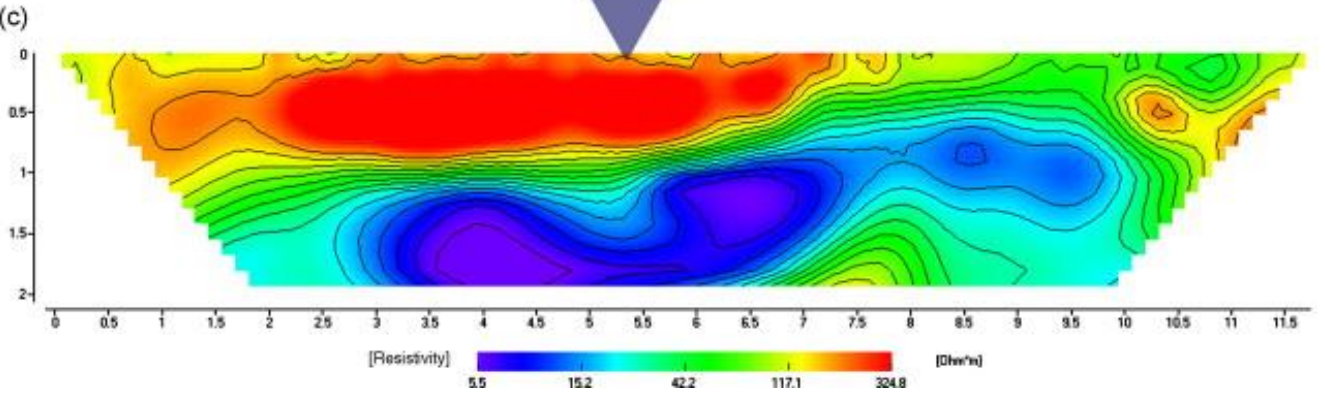

Figure 10: Data acquisition and processing in ERT; (a) a linear array of electrodes with two quadrupoles at minimum spacing (top) and one quadrupole at maximum spacing (bottom). Dots represent electrodes and full triangles represent the centre of soil volumes measured by the corresponding quadrupole; (b) soil apparent resistivity 2D pseudosection obtained after data acquisition; (c) soil resistivity 2D section obtained after data inversion with numerical modelling (Amato, et al., 2009) 
471 ERT has been widely applied for detecting soil compaction (Besson, et al., 2004), water

472 content and flow in soil and plants (Loperte, et al., 2006), soil cracks (Samouelian, et al., 473 2005) and tillage effects (Basso, et al., 2010). The plant root zone shows variations in soil 474 electrical resistivity (Panissod, et al., 2001), and resistive soil volumes have been 475 correlated to large tree root structures (Amato, et al., 2008) (Zenone, et al., 2008).

476 Amato, et al (2008) conducted research in which the root biomass of alder trees was 477 accurately mapped in 2D. This study demonstrated that the use of ERT for the non478 destructive characterisation of root systems' spatial structure could reduce the 479 coefficient of variability of root measurements, which is more significant than that of 480 above-ground plant parts (Amato \& Ritchie, 2002).

481 A quantitative relationship between the electrical resistivity of the soil and the biomass 482 of the roots has been widely demonstrated (Loperte, et al., 2006) (Amato, et al., 2008). 483 However, in the case of low root biomass densities, the electrical response of the roots 484 is indistinguishable from the background noise. In fact, it is assumed that it is of the same 485 order of magnitude as the response coming from the other characteristics of the soil, 486 and consequently too weak to be detected (Amato, et al., 2009).

The main advantage of this technique is that it is totally non-destructive, as it does not 488 disturb the structure nor the functioning of soil. Subsurface heterogeneities can be determined, in one, two or three dimensions, both non-invasively and dynamically 490 (Samouelian, et al., 2005). Variations in time of root systems can be obtained, and 491 different and more detailed information can be obtained by varying the operating configurations or the distance between the electrodes, depending on soil properties. 493 Furthermore, this methodology has a low application cost, and can be applied on a large 494 scale.

However, this investigation technique can be influenced by several factors, which could potentially act at the same time, making interpretation of the results difficult. 497 Systematic errors can result from poor electrode contact or noise averaging, although 498 these can be avoided by carrying out replicated and reciprocal measurements (positive and negative current and potential electrodes reversed) (Samouelian, et al., 2005). 
500

501

502

503

504

505

506

507

508

509

510

511

512

513

514

515

516

517

Moreover, ERT field investigations should be coupled with laboratory studies, to calibrate the resistivity against different soil conditions. (Samouelian, et al., 2005).

\subsubsection{Acoustic detection}

The acoustic detection of wood is widely used for tree investigations, ranging from the detection of decay, cracks, hollows or holes (Buza \& Goncz, 2015) (Wang, et al., 2007) (Grabianowski, et al., 2006) to material characterisation for wood evaluation and quality assessment (Bucur, 2006). Therefore, the acoustic detection of roots has been tested, based on the difference of velocity in wood and soil. In fact, the velocity of the acoustic signal in soil is between $250-400 \mathrm{~m} / \mathrm{s}$, depending on soil type and moisture content, while the velocity in wood is between 2000 and 4000 m/s (Bucur, 2006) (Buza \& Goncz, 2015).

The device for acoustic measurements consists of a transmitter, a receiver, and a timemeasuring component. The transmitter is needle-like and must be placed onto the trunk at ground level, while the receiver is a long metal spike (30 cm or longer), which has a suitable coupling for the soil (Figure 11) (Buza \& Goncz, 2015). During an investigation, the transmitter sends a very short signal, which is then reflected and read by the receiver. The presence of roots decreases the travel time significantly, making it possible to locate them.

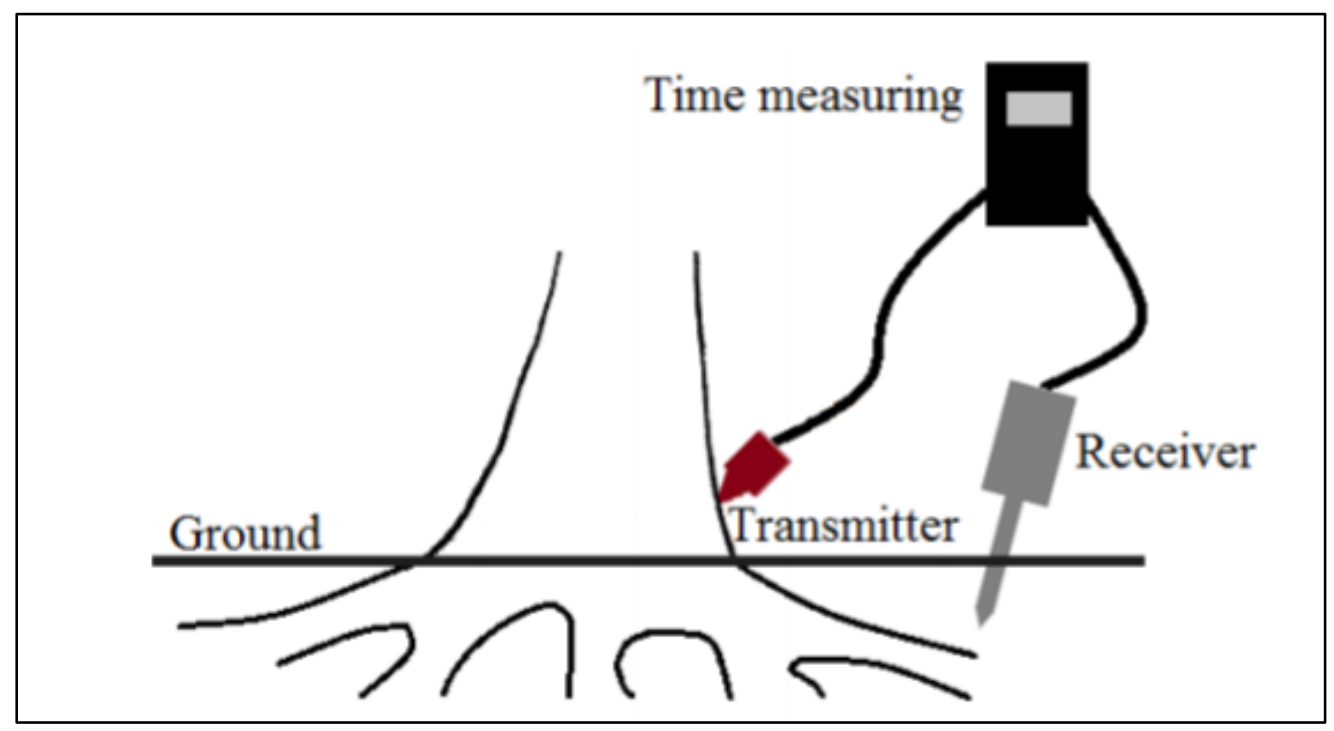


520 Using this technique, it is possible to identify roots with a diameter of $4 \mathrm{~cm}$ upwards,

521 with a maximum depth of investigation of $50 \mathrm{~cm}$. Furthermore, it is possible to separate

522 two roots from each other if they are at least $20 \mathrm{~cm}$ apart (Buza \& Divos, 2016). These

523 achievements are limitations as well, as the detection of small or deep roots is not

524 possible. Furthermore, research carried out by Iwase, et al. (2015) demonstrated that

525 the signal is highly sensitive to water content. Finally, other buried objects, such as rocks,

526 can disguise the signal, making it difficult to recognise root system architecture correctly

527 (Divos, et al., 2009). Given that this methodology, despite the promising results, is still

528 in its infancy, it is often coupled with other NDT methods, in order to further investigate

529 its potential (Buza \& Goncz, 2015).

530

\subsubsection{X-ray computed tomography}

531

$\mathrm{X}$-ray computed tomography $(\mathrm{CT})$ is a non-destructive, non-invasive technique that can

532 be used to visualise the interior of objects in 2D and 3D based on the principle of attenuation of an electromagnetic wave. X-ray CT has been repeatedly demonstrated to be an efficient methodology for imaging and studying soil systems. CT uses X-rays to obtain cross-sectional images of an object, which contain information regarding the attenuation of the X-rays, a function of the density of the sample material (Mahesh, 2002). These slices are then reconstructed to provide a 3-D visualisation of the sample volume.

539 During CT acquisition, $\mathrm{X}$-rays are produced in a highly evacuated tube, which contains 540 an anode, usually platinum or tungsten, and a cathode (Wildenschild, et al., 2002). When

541 a high voltage is applied across these electrodes, accelerated electrons produce $\mathrm{X}$-rays

542 as they strike the anode. As the X-ray beams pass through a sample, the object itself

543 becomes a secondary source of X-rays and electrons. A portion of the primary incident 544 beam is therefore absorbed or scattered. This reduction in intensity of the X-ray as it 545 passes through the investigated object is called attenuation. The beam is projected onto 546 the detector, which measures the change in energy intensity (Mooney, et al., 2012).

547 X-ray CT offers great potential for examining undisturbed root systems architecture in 548 soils, and its potential has been widely investigated within the last decades (Heeraman, 
549 et al., 1997) (Gregory, et al., 2003). The imaging of plant roots in soil using X-ray CT relies

550 on sufficient contrast in X-ray attenuation between growth medium solids, air-filled

551 pores, soil water, plant material and organic matter. The attenuation of these materials

552 varies with several factors including soil type, soil moisture content, the proximity of

553 roots to organic matter or air-filled pores and root water status (Kaestner, et al., 2006).

554 The limitations of this technique are the overestimation of root diameter during image

555 analysis due to the proximity of water and air within the soil (Perret, et al., 2007), and

556 the underestimation of root length and number of lateral roots due to the fact that root

557 material cannot be easily distinguished from other soil components. To minimise the

558 effects of similar attenuation between the soil and plant fractions, researchers have

559 focused on plants with coarse roots (Hargreaves, et al., 2009), artificial soil systems

560 (Perret, et al., 2007), manipulating the water content of the sample and undertaken

561 convoluted image processing to enhance contrast. Still, it is difficult to distinguish the

562 boundaries between adjacent structures (Mooney, et al., 2012).

563 Advancements in CT technology include a reduction in scan and reconstruction times by 564 at least an order of magnitude, automated algorithms to remove artefacts and more 565 sophisticated detectors that have significantly increased the raw scan image quality 566 (Mooney, et al., 2012). Research is now focused on investigating this technique's future 567 potential in terms of the interaction between roots and their soil environment (Tracy, et 568 al., 2010). 


\section{Ground Penetrating Radar}

570 Ground penetrating radar (GPR) is a non-destructive testing method used to detect 571 changes in physical properties within the shallow subsurface (Daniels, 1996). The 572 operating principles of a GPR system are based on the theory of electromagnetic (EM) 573 fields, which is described by Maxwell's equations (Jol, 2008). In addition, GPR 574 effectiveness relies on the response of the investigated materials to the EM fields, which 575 is ruled by the constitutive equations (Jol, 2008). Therefore, the combination of the EM 576 theory with the physical properties of the material is essential for a quantitative 577 description of the GPR signal.

\subsection{GPR theoretical background}

579 A standard GPR system consists of three essential components: a control unit (including 580 a pulse generator, computer, and associated software), antennas (including paired 581 transmitting and receiving antennas), and a display unit (Guo, et al., 2013) (Figure 12).

582 During a GPR investigation, the transmitting antenna generates short impulses of EM 583 energy, which are launched into the investigated medium where they propagate as 584 waves (Daniels, 1996). When these waves hit a target with different electrical or 585 magnetic properties, reflections are generated, which are then diffracted back towards 586 the surface and recorded by the receiving antenna. The remaining energy, conversely, 587 continues to travel into the medium until it is completely attenuated (Daniels, 1996). 588 The control unit samples and filters the collected information, and then combines it into 589 a reflection trace (also named A-scan), recording the time between the emission of the 590 reflected signal and its reflection on the vertical axis and the amplitudes of the received 591 signals on the horizontal axis (Daniels, 2004). Being an individual trace, the A-scan 592 provides punctual information about the subsurface configuration (Benedetto, et al., 593 2017). 


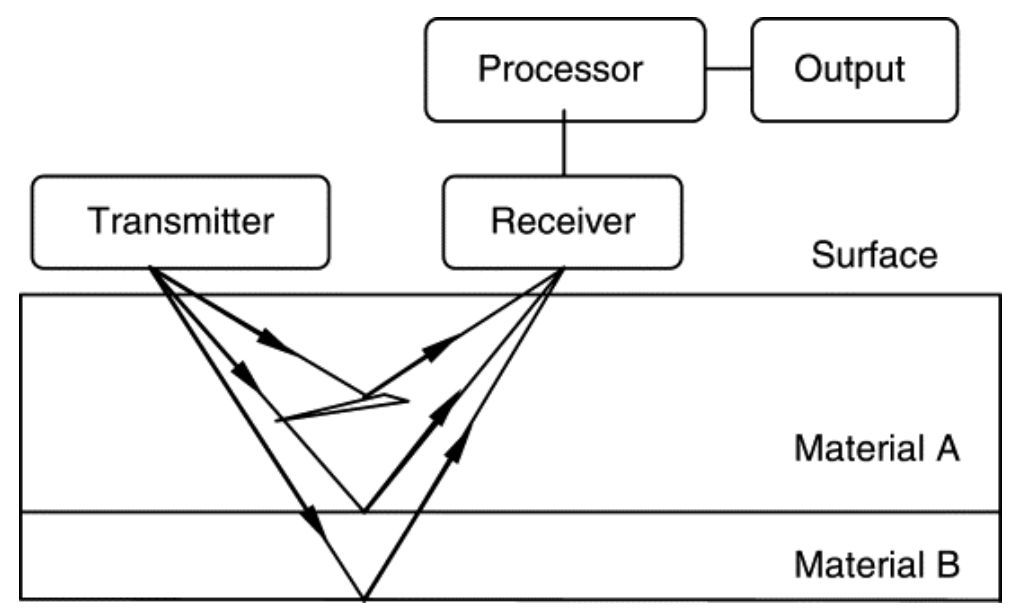

595

Figure 12: GPR operating principles

596 The depth of a target can be derived from the propagation velocity $(V)$, as follows 597 (Daniels, 1996):

$$
D=\frac{V \times t}{2}
$$

598 where $D$ in the depth and $t$ is the two-way travel time. Instead, wave velocity can be 599 calculated from the following equation (Lorenzo, et al., 2010):

$$
V=\frac{1}{\sqrt{\frac{\mu \varepsilon}{2}\left(\sqrt{1+\left(\frac{\sigma}{\omega \varepsilon}\right)^{2}}\right)+1}}
$$

600 where

601

- $\mu$ is the magnetic permeability;

602

- $\sigma$ is the electrical conductivity;

603

- $\varepsilon$ is the dielectric permittivity;

604

- $\omega$ is the angular frequency ( $\omega=2 \pi f$, where $f$ is frequency) of the emitted

605 pulse.

606

A formula for the estimation of propagation velocity for low conductive and 607 nonmagnetic materials $\left(\sigma \ll \omega \varepsilon\right.$ and $\mu_{r}=1$, where $\mu_{r}$ is the relative magnetic 608 permeability) has also been proposed (Jol, 2008) (Daniels, 2004): 


$$
V=\frac{1}{\sqrt{\mu \varepsilon}}=\frac{c}{\sqrt{\varepsilon_{r}}}
$$

609 where

- $\quad c$ is the speed of light in vacuum (0.2998 $\mathrm{m}$ per nanosecond);

611

- $\varepsilon_{r}$ is the relative dielectric permittivity.

612 The reflected energy amplitude at an interface between two materials depends on the 613 reflection coefficient $R$ (al Hagrey, 2007):

$$
R=\frac{\sqrt{\varepsilon_{r 1}}-\sqrt{\varepsilon_{r 2}}}{\sqrt{\varepsilon_{r 1}}+\sqrt{\varepsilon_{r 2}}}=\frac{V_{2}-V_{1}}{V_{1}+V_{2}}
$$

614 where

$615-\varepsilon_{r 1}$ is the relative dielectric permittivity of the overlying material;

616

- $\varepsilon_{r 2}$ is the relative dielectric permittivity of the underlying material;

$617-V_{1}$ is the propagation velocity in the overlying material;

618

- $V_{2}$ is the propagation velocity in the underlying material.

619 During a survey, GPR is moved along a detection transect, and EM pulses are generated 620 at a specified interval of time or distance. As reflected signals are recorded, traces can 621 be integrated into a radargram (also called B-scan) that allow for a 2D representation of 622 the subsurface (Figure 13). The B-scan mode is a widely used imaging methodology, as 623 it permits to visualise the presence of buried objects (Bianchini Ciampoli, et al., 2019).

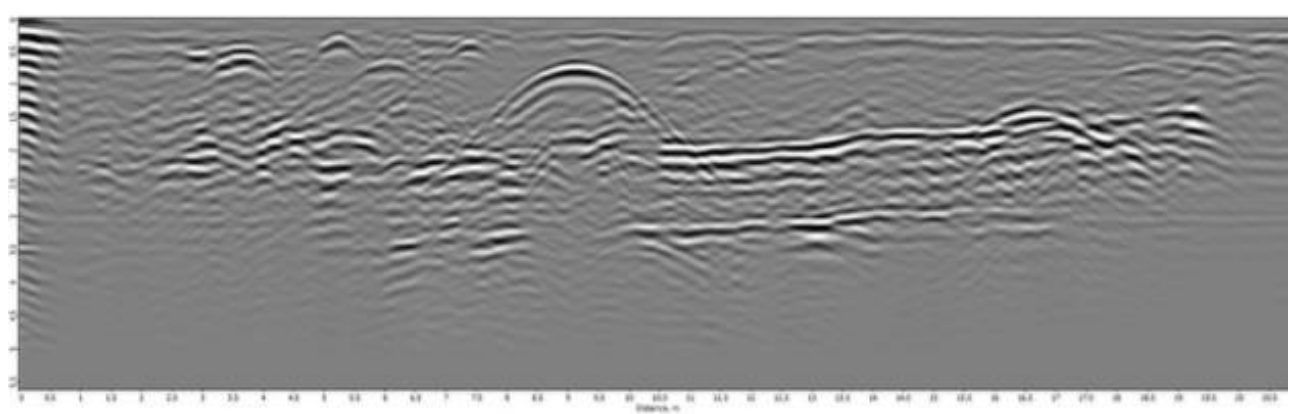

625 Figure 13: A typical radargram or Bscan 
626 The GPR transmitting antenna produces energy in the form of a beam that penetrates

627 into the ground in the form of an elliptical cone. As the propagation depth increases, the

628 cone radius also expands, resulting in a larger footprint scanned beneath the antenna

629 (Figure 14a). The footprint area can be approximated by the formula (Conyers, 2002):

$$
A=\frac{\lambda}{4}+\frac{D}{\sqrt{\varepsilon_{r}+1}}
$$

630 where

$631 \quad-A$ is the long dimension radius of footprint;

$632 \quad-\lambda$ is the centre frequency wavelength of radar energy;

$633-D$ is the depth from the ground surface to the reflection surface;

$634-\varepsilon_{r}$ is the average relative dielectric permittivity of scanned material from the

635 ground surface to the depth of reflector $(D)$.

636 Based on this feature of propagating waves, radar energy will therefore be reflected 637 before and after the antenna is positioned above a buried object. As the antenna moves 638 closer to the object, the recorded two-way travel time decreases, while when the 639 antenna moves away from it, the same phenomenon is repeated conversely, generating 640 a reflection hyperbola, the apex of which indicates the exact location of the buried 641 object (Guo, et al., 2013) (Figure 14b). 
642

643

644

645

646

647

648

649

650

651

652

653

654

655

656

657

658

659

660

661

662

663

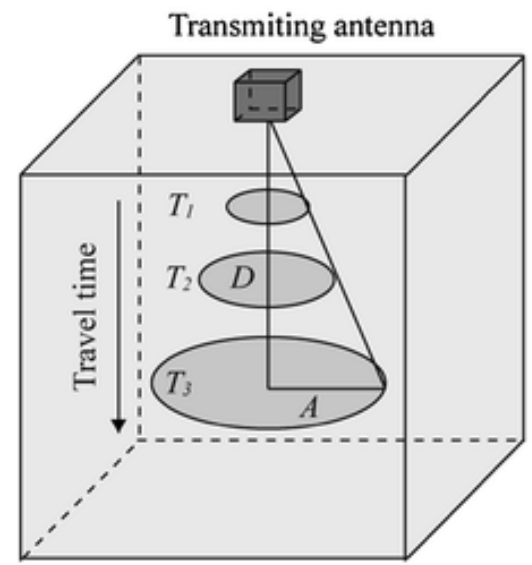

a

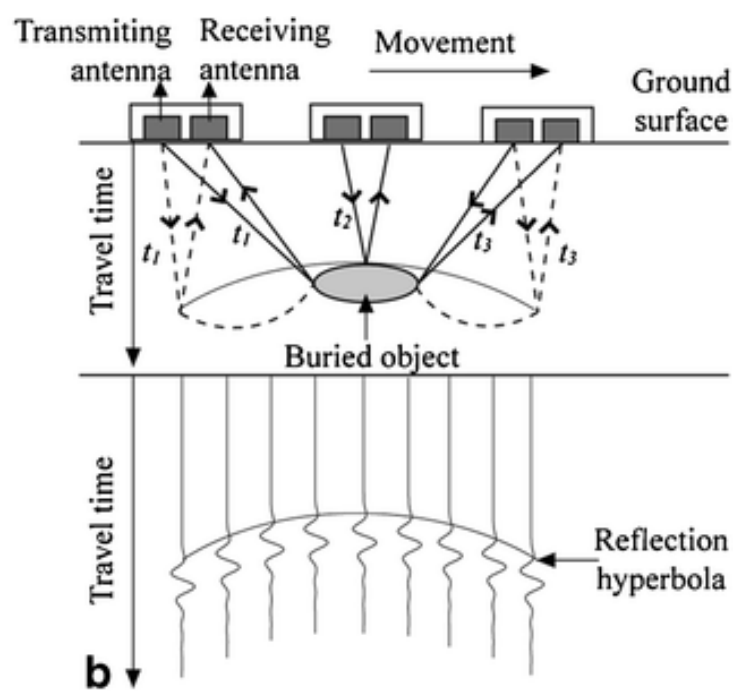

Figure 14: Schematic illustration of the conical radiating pattern of GPR waves and generation of a reflection hyperbola (Guo, et al., 2013): a) development of a footprint with increasing travelling time; b) detection of a buried object with the creation of a reflection hyperbola

The GPR resolution, and therefore its capability to discriminate between two closely spaced targets as well as the minimum size detectable, correlates negatively with the footprint area. GPR detection resolution depends on the antenna frequency, the EM properties of the medium, and the penetrating depth (Hruska, et al., 1999). Therefore in a survey, the selection of the appropriate GPR features, including frequency operations, the type of antenna or its polarization rely on a number of factors, such as the size and shape of the target and the transmission properties of the investigated medium, as well as the characteristics of the surface (Daniels, 2004).

Advances in GPR data processing and visualisation software have allowed for the creation of 3D pseudo-images (also called C-scans) of the subsurface, obtained by interpolating multiple 2D radargrams. A C-scan provides an amplitude map at a specific time (or depth) of collection (Benedetto, et al., 2017), and is therefore helpful in visualising a trend of the amplitude values all over the investigated domain.

In regard to GPR data processing and analysis, appropriate signal processing techniques are needed to provide easily interpretable images to operators and decision-makers (Daniels, 2004). Most of the techniques that are applied today originate from seismic theory (Benedetto, et al., 2017), as both disciplines involve the collection of pulsed signals in the time domain. It is not possible to establish a unique methodology, as it 
depends on the purpose of the survey, the features of the used radar and the conditions of the investigated medium. Furthermore, the analysis of GPR data is a challenging issue, as the interpretation of GPR data is generally non-intuitive and considerable expertise is therefore needed.

\subsection{GPR applications in the assessment of tree root systems}

GPR has been employed for many applications and in several disciplines, such as archaeological investigations (Goodman, 1994), bridge deck (Alani, et al., 2013) and tunnel analyses (Alani \& Tosti, 2018), the detection of landmines (Potin, et al., 2006), civil and environmental engineering applications (Tosti et al., 2018b) (Benedetto, et al., 2017) (Benedetto, et al., 2015) (Loizos \& Plati, 2007), and planetary explorations (Tosti \& Pajewski, 2015), for about forty years.

Although GPR has commonly been used to characterise soil profiles (Lambot, et al., 2002) (Huisman, et al., 2003), roots have often been considered an unwanted source of noise that usually complicates radar interpretation (Zenone, et al., 2008). However, over the past decade, GPR has been increasingly used for tree root assessment and mapping, as it is completely non-invasive and does not disturb the soils or bring harm to the examined trees or the surrounding environment. For these reasons, repeated measurements of root systems are possible, allowing for the study of the roots' developmental processes.

The first application of GPR that relates to the mapping of tree root systems dates back to 1999 (Hruska, et al., 1999). In this study, a GPR system with a central frequency of $450 \mathrm{MHz}$ was employed to map the coarse roots of 50-year-old oak trees, and measurements were made in two directions within a $6 \mathrm{~m}$ by $6 \mathrm{~m}$ square, with a $0.25 \mathrm{~m}$ $x 0.25 \mathrm{~m}$ profile grid, at $0.05 \mathrm{~m}$ intervals. After data processing, the root system of the large oak tree was analysed in detail by applying depth correlations of GPR indications from single profiles to develop a 3D picture. Additionally, the root system was excavated and photographed, and root lengths and diameters were measured to verify the radar data. The researchers confirmed that the resolution of the GPR system was sufficient to distinguish the roots that were $3 \mathrm{~cm}$ to $4 \mathrm{~cm}$ in diameter. Diameters of roots detected 
693

694

695

696

697

698

699

700

701

702

703

704

by the GPR system corresponded to measured diameters of excavated roots with an error of between 1 and $2 \mathrm{~cm}$. The GPR system determined the length of individual roots, from the stem to the smallest detectable width, with an error margin of about $0.2 \mathrm{dm}$ to $0.3 \mathrm{dm}$. Higher frequencies together with smaller measurement intervals were applied, and this method improved the resolution and accuracy to less than $1 \mathrm{~cm}$. In conclusion, the researchers claimed to have successfully tested GPR in a forest and woodland environment, where the soil is relatively homogenous. The output of this study was criticised several years later (Guo, et al., 2013), because the 3D views of the coarse root system were redrawn manually based on the GPR radargram, but no specific information was provided regarding how it had been done (Figure 15). Assuming that the maps were redrawn arbitrarily according to the operator's personal experience, bias may therefore have been introduced.

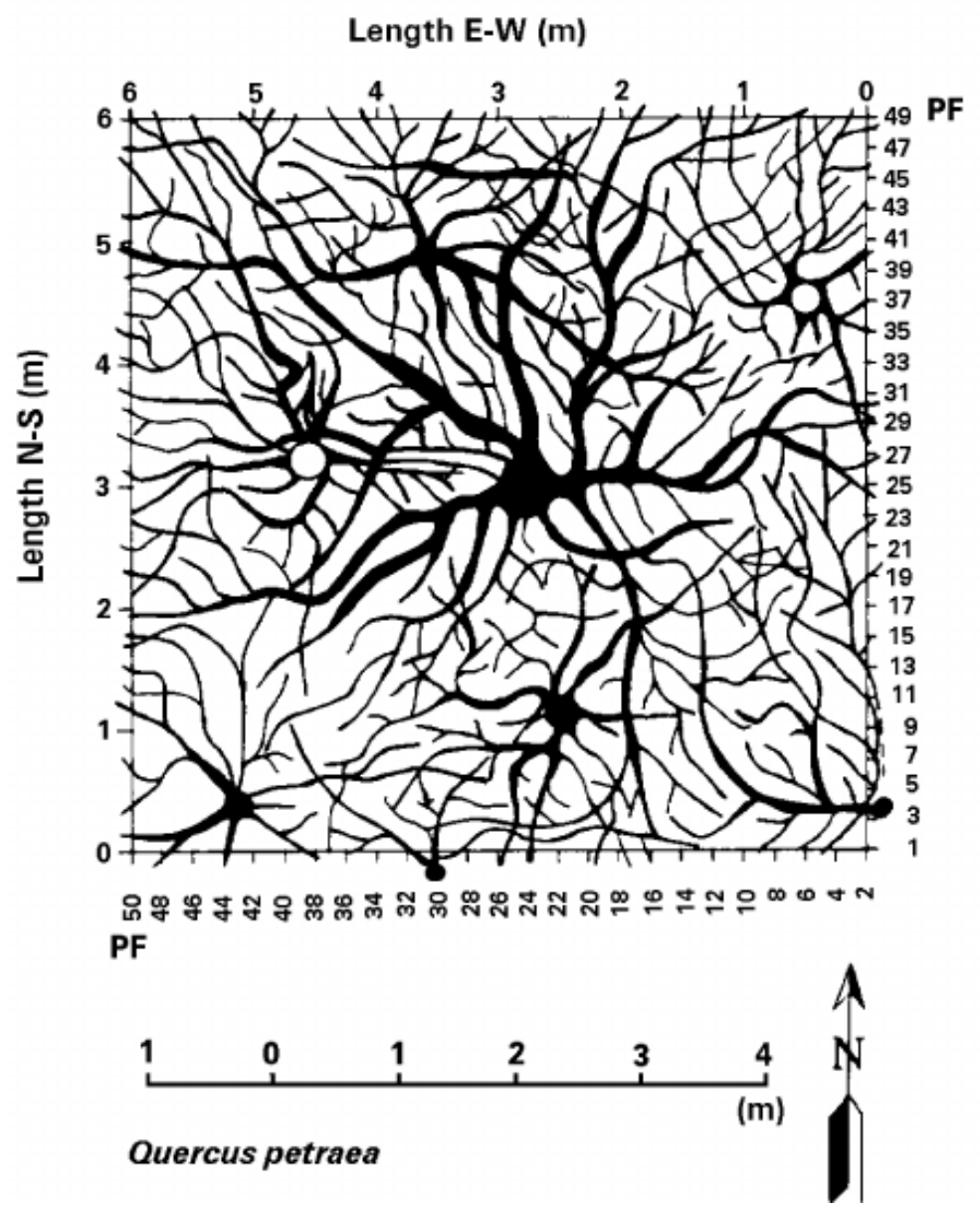

Figure 15: Hand-drawn reconstruction of a tree root system based on the analysis of GPR data (Hruska, et al., 1999) 
Attempts to map tree root systems have continued throughout the years (Sustek, et al., 1999) (Cermak, et al., 2000) (Wielopolski, et al., 2000), with alternate and controversial results. The most significant barrier to mapping complete root systems with GPR is the inability to distinguish individual roots when tight clusters of roots are encountered, as they give one only large parabolic reflection (Butnor, et al., 2001). Furthermore, many pieces of research were carried out under controlled conditions (Barton \& Montagu, 2004), therefore limiting the significance of the results for in situ tree root mapping. Moreover, the minimum detectable size for tree roots is still a subject of discussion. In fact, tests conducted under controlled conditions confirmed that it was possible to detect fine roots ( $0.5 \mathrm{~cm}$ in diameter or less) (Butnor, et al., 2001), while tests carried out in the field demonstrated that only coarse roots with diameters greater than $5 \mathrm{~cm}$ could be identified (Ow \& Sim, 2012).

Furthermore, research has concentrated on the use of GPR as an appropriate tool for use on valuable trees, or trees in situations where excavation is not possible, such as growing near pavements, roads, buildings or on unstable slopes (Stokes, et al., 2002). GPR data were able to reliably locate roots under pavements and provided a reasonably accurate root count in the compacted soil under concrete (Bassuk, et al., 2011) and asphalt (Cermak, et al., 2000). This is possible thanks to the difference in water content between roots and soil, which can provide the necessary permittivity contrast and therefore allow root detection by GPR (Wielopolski, et al., 2000). Also, it facilitates the distinction between roots and buried utilities (i.e. cables and pipes), which could otherwise generate signal interference, affecting the GPR survey (Ow \& Sim, 2012).

Another testing issue that has been investigated is the survey methodology. Two experimental sites situated in Italy, subject to different climates and hydrological conditions, were investigated for this purpose (Zenone, et al., 2008). In this study, GPR measurements were taken using antennas of 900 and $1500 \mathrm{MHz}$ applied in square and circular grids (Figure 16): even though square grids are preferable for GPR lines, results obtainable with circular transects (created by rotating the GPR around the tree, keeping a constant radial distance) were tested to ensure a quasi-perpendicular scanning of root 

was challenging to push a radar system in circles over roots and stones.

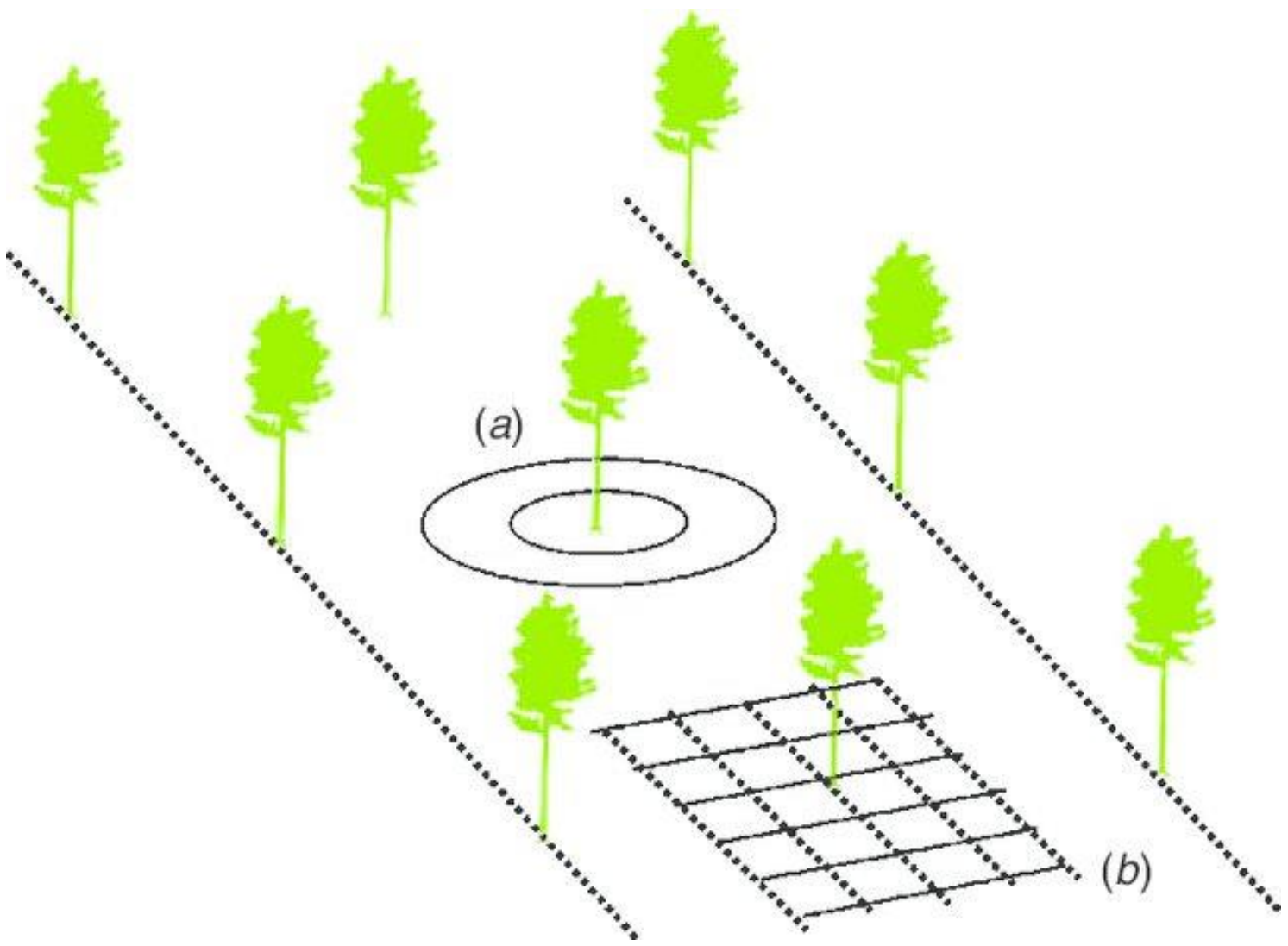

Figure 16: GPR setups for tree root system survey using a) circular transects and b) square grids (Zenone, et al., 2008)

742 Most of the aforementioned methodologies tested the reliability of their results by 743 digging or uprooting the investigated trees. Zenone, et al. (2008) excavated the root 744 system with an air-spade and pulled it out using a digger; a laser measurement system 745 was then applied in order to create a scan, and the 3D root system architecture was 746 reconstructed.

747 A comparison between the laser scan point cloud and the sections of GPR scans (Figure 748 17) returned a limited grade of correspondence, and the authors stated that this might 749 be due to an alteration of the root system architecture that occurred during the 750 excavation. Nevertheless, the use of GPR for 3D coarse root system architecture reconstruction was further criticised (Guo, et al., 2013). 
752

753

754 Set aside the recognition and mapping of tree roots, a challenge that is still object of 755 discussion is the quantification of the biomass of tree roots. As it is widely 756

757

758

759

760

761

762

763

764

765

766

767

768 acknowledged, the estimate of tree root mass density is crucial for the evaluation of the health status of the tree, for the stability of the tree itself and the stability of the soil, as tree roots are used for the reinforcement of slopes. Not least, root mass evaluation is essential for understanding the storage of carbon in the ecosystem (Stover, et al., 2007).

Traditional methods for estimating root biomass are usually destructive, timeconsuming and expensive, as well as often inaccurate (Birouste, et al., 2014). The application of NDT methods in this research area is still at the early stage, and the achieved results are still not accurate enough (Aulen \& Shipley, 2012).

GPR has proven to be efficient in the estimation of coarse root biomass (Guo, et al., 2013). Several studies have been conducted so far in field conditions (Butnor, et al., 2001) (Butnor, et al., 2003) (Stover, et al., 2007) (Butnor, et al., 2008) (Samuelson, et al., 2008) (Borden, et al., 2014) and in laboratory environment (Cui, et al., 2011). GPR has shown potential for root quantification, as coarse root biomass has been assessed with

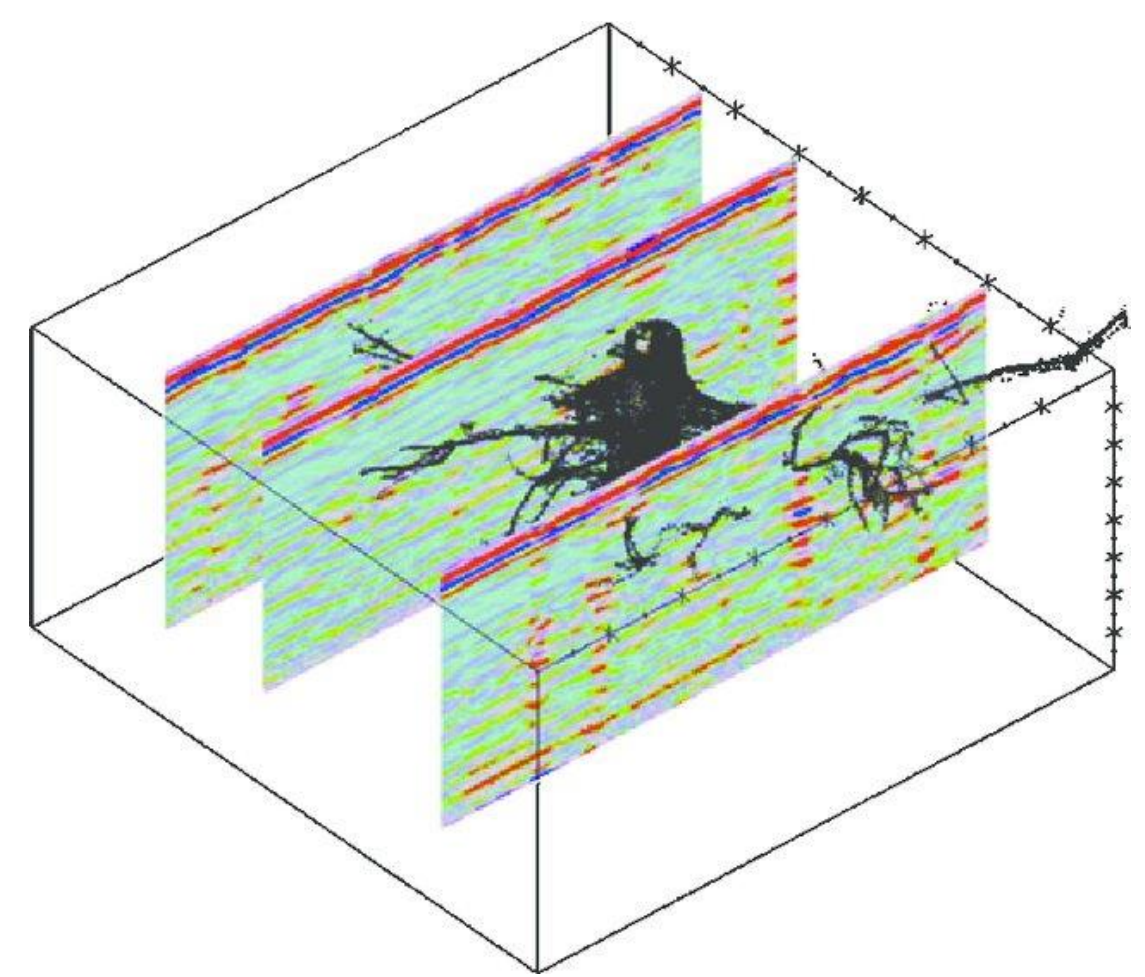

Figure 17: Comparison between 3D rendering from a laser scanner and GPR Bscans (Zenone, et al., 2008) 
769 reasonably good accuracy (Guo, et al., 2013). However, uncertainty still affects the 770 precision of the existing methodologies. Currently, a limiting factor for a correct root 771 density estimation is the root water content which, if too low, can lead to an 772 underestimation of root biomass (Guo, et al., 2013).

773 In conclusion, all the above-mentioned NDT methods have proven viability in the 774 assessment of tree root systems. However, the knowledge of the application of some of 775 these techniques in tree assessment is still in its infancy. Moreover, their employment 776 can be troublesome, as the required equipment is often difficult to operate. In addition, 777 the application of these methods can often be very expensive. On the other hand, GPR 778 is gaining attention in view of the high versatility, the rapidity of its data collection and 779 the provision of reliable results at relatively limited costs. It has also proven to be a 780 reliable instrument for the assessment of tree root systems. The advantages and 781 limitations of the aforementioned ND techniques in the assessment of tree root systems 782 are summarised in Table 2. 


\begin{tabular}{|c|c|c|c|c|c|}
\hline Working principle & Method & $\begin{array}{c}\text { Characteristic } \\
\mathrm{s}\end{array}$ & Applications & Advantages & Limitations \\
\hline Imaging & (Mini)Rhizotrons & $\begin{array}{l}\text { Non- } \\
\text { destructive } \\
\text { Slightly } \\
\text { invasive }\end{array}$ & $\begin{array}{l}\text { Quantification of fine root } \\
\text { growth }\end{array}$ & $\begin{array}{l}\text { - } \quad \text { High-resolution imaging } \\
\text { - } \quad \text { Frequent inspections }\end{array}$ & $\begin{array}{ll} & \text { Modification of soil hydrology and physics } \\
\text { - } & \text { Only small portions of the root system can be observed } \\
\text { - } & \text { Disparity in results obtained from different image processing } \\
\text { - } & \text { methods } \\
\text { - } \quad \text { Expensive of installation } \\
\text { - Impossible to install in certain environments (i.e. urban trees) }\end{array}$ \\
\hline Mechanical & Pulling test & $\begin{array}{l}\text { Non- } \\
\text { destructive } \\
\text { Invasive }\end{array}$ & $\begin{array}{l}\text { Assessment of tree root plate } \\
\text { stability }\end{array}$ & $\begin{array}{l}\text { - } \quad \text { Provides a safety factor for tree stability } \\
\text { - Test of the elastic response of the tree trunk }\end{array}$ & $\begin{array}{ll} & \text { Invasive } \\
\text { - } & \text { Not completely realistic (i.e. cannot simulate wind effects) } \\
\text { - } & \text { Affected by temperature conditions } \\
\text { - } & \text { Not useful for understanding the causes of tree instability }\end{array}$ \\
\hline Electrical & ERT & $\begin{array}{l}\text { Non- } \\
\text { destructive } \\
\text { Non-invasive }\end{array}$ & $\begin{array}{l}\text { Detection of root distribution } \\
\text { Quantification of root } \\
\text { biomass }\end{array}$ & $\begin{array}{ll}\text { - } & \text { Easiness of data collection } \\
\text { - } & \text { Suitable for measurements repeated over } \\
\text { - } & \text { Various scales application } \\
\text { - } & \text { Possibility of } 1 D, 2 D \text { and 3D surveys } \\
\text { - } & \text { Depth of detection }\end{array}$ & $\begin{array}{l}\text { - } \quad \text { Systematic errors due to poor electrode contact } \\
\text { - } \quad \text { Long measurement times } \\
\text { - } \quad \text { Non-uniqueness of the solution in the inversion scheme } \\
\text { - Difficult to discern the effect of roots from the background noise } \\
\text { for low root biomass }\end{array}$ \\
\hline Acoustic & $\begin{array}{l}\text { Acoustic } \\
\text { detection }\end{array}$ & $\begin{array}{l}\text { Non- } \\
\text { destructive } \\
\text { Slightly } \\
\text { invasive }\end{array}$ & Detection of roots & - Successful detection of coarse roots & $\begin{array}{ll}\text { - } & \text { Small roots (diameter }<4 \mathrm{~cm} \text { ) are not detected } \\
\text { - } & \text { Superficial depth of detection }(<50 \mathrm{~cm}) \\
\text { - } & \text { High sensitivity to water content } \\
\text { - } & \text { Difficult to discern roots from other buried objects }\end{array}$ \\
\hline Electromagnetic & X-ray CT & $\begin{array}{l}\text { Non- } \\
\text { destructive } \\
\text { Non-invasive }\end{array}$ & $\begin{array}{l}\text { 3D mapping of roots } \\
\text { Quantification of root length } \\
\text { and diameter }\end{array}$ & $\begin{array}{ll}\text { - } & \text { High-resolution imaging } \\
\text { - } & \text { Suitable for measurements repeated over } \\
\text { - } & \text { Detection of fine roots }\end{array}$ & 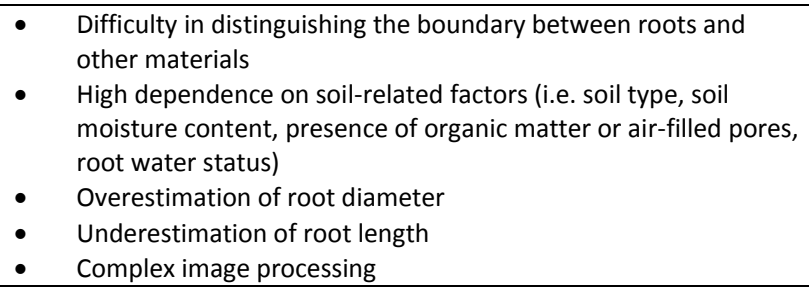 \\
\hline
\end{tabular}




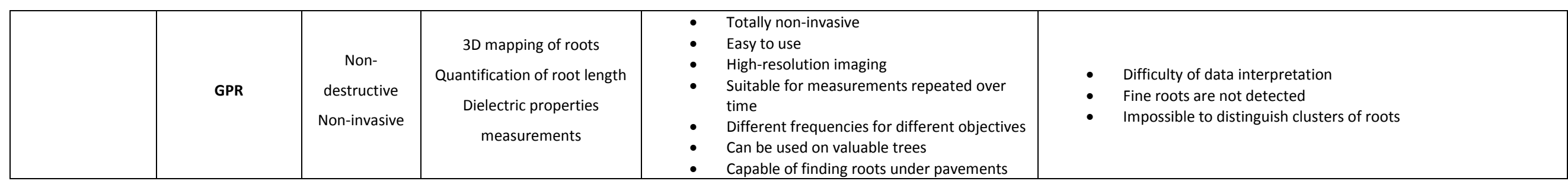


6. New methodological and data processing prospects for the assessment of tree root systems architecture using Ground Penetrating Radar: a case study

Recent advances in tree root mapping using GPR have led to the reconstruction of root system geometry using correlation analysis in the 3D domain (Alani, et al., 2018). In this study, two trees of different species, fir and oak, were investigated using circular and semi-circular scanning configurations, in order to test the viability of a novel technique for the creation of a three-dimensional root system model.

This study was further developed by Lantini, et al. (2018), with the aim of assessing interactions between different tree root systems. Interconnections between different root systems allow the transmission of pathogenic diseases and fungi. Research into how these roots interact with each other and with the surrounding environment is essential for the achievement of effective containment practices. To achieve this aim, this pilot research study focused on the estimate of root mass density, and this objective was addressed by evaluating the total root length per reference unit. Promising results were obtained, demonstrating that local increases in density occur in the area where interconnections are supposed to happen.

802

Further research, which includes advanced signal processing, is now under development, with the aim of reducing uncertainty and false alarms in root detection. To this extent, a case study is presented, in which a dedicated data processing methodology, based on three main chronological stages, is applied to GPR data. An improved pre-processing algorithm is proposed, with the aim of reducing clutter in raw GPR data, improve target detection and increase deeper reflections which are likely to be related to deep root systems but have been attenuated due to increasing depths or highly conductive materials. Furthermore, advanced signal processing techniques are applied, in an effort to remove ringing noise from GPR data and focus on the response

811 from the target. Subsequently, an iterative procedure for tree root recognition and

812 tracking and root system architecture reconstruction in a 3D domain is implemented, based on a correlation analysis between identified targets. Lastly, the domain is divided 
814 into reference volume units and root density maps are produced. This approach has

815 given promising results, proving that GPR has the potential to identify both the shallow

816 (within the first $25 \mathrm{~cm}$ of soil) and the deep (more than $25 \mathrm{~cm}$ from the soil surface) root

817 systems, and find viable root paths, allowing for the construction of three-dimensional

818 models of root systems for different species of trees.

819

\subsection{Materials and methods}

820

\subsubsection{The survey technique}

821 The survey was carried out in Walpole Park, Ealing, London (United Kingdom). The soil

822 around a mature tree (trunk circumference at ground level of $3.83 \mathrm{~m}$ and radius of 0.61

$823 \mathrm{~m}$ ) was investigated (Figure 18). 24 circular scans were performed on the soil around the

824 tree trunk, starting $0.50 \mathrm{~m}$ from the bark and then $0.30 \mathrm{~m}$ apart from one another. Thus,

825 an overall area of $197.69 \mathrm{~m}^{2}$ was examined.

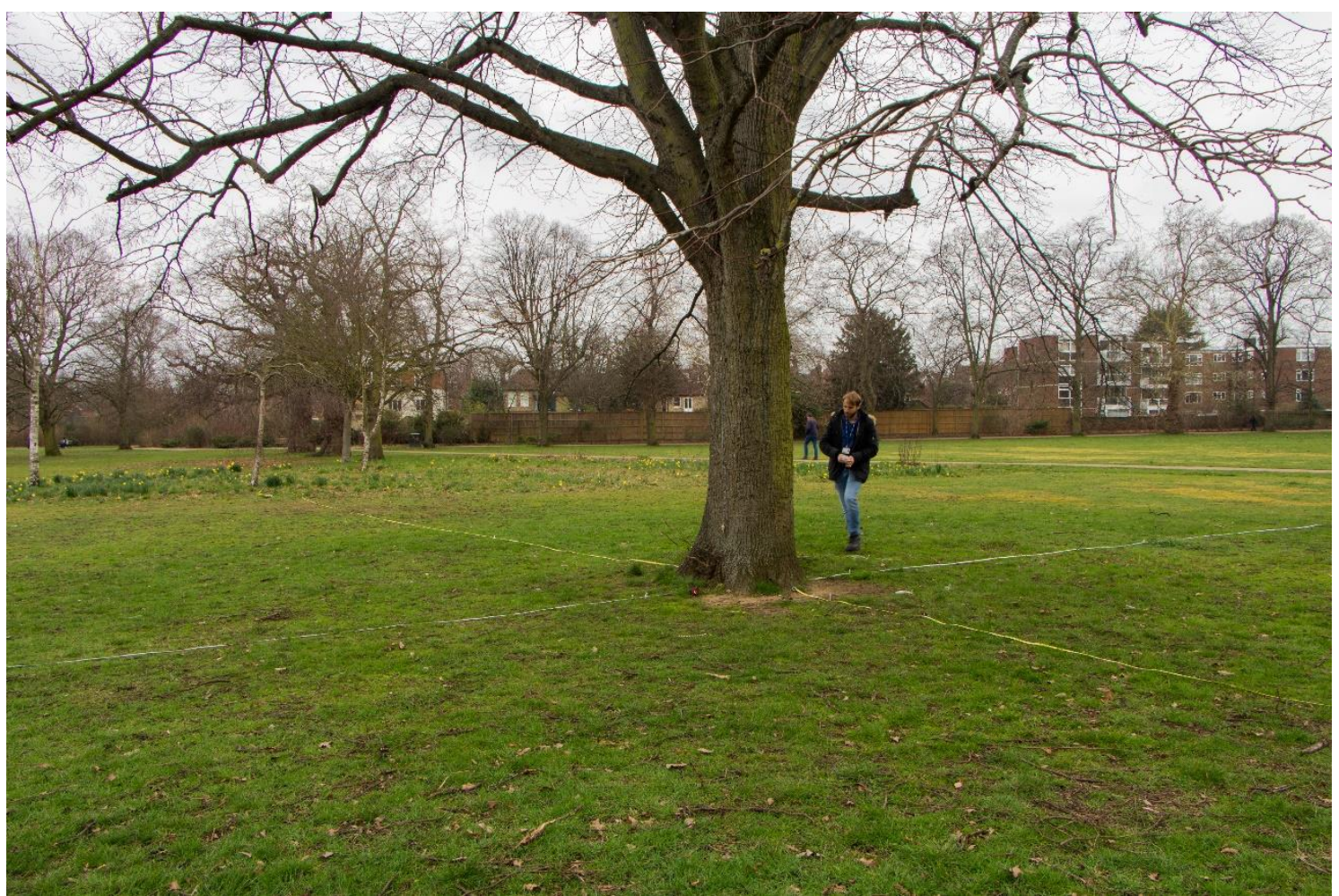

827

Figure 18: The investigated area

828 
830 The survey was performed using a ground-coupled GPR system (Opera Duo, IDS

831 GeoRadar (Part of Hexagon)), equipped with $700 \mathrm{MHz}$ and $250 \mathrm{MHz}$ central frequency

832 antennas (Figure 19). Data acquisition was performed using a time window of 80 ns and

833512 samples. The horizontal resolution was set to $3.2 \times 10^{-2} \mathrm{~m}$. For this study, only data

834 from the $700 \mathrm{MHz}$ frequency antenna were analysed, as these provide the highest

835 effective resolution (Benedetto, et al., 2011) (Benedetto, et al., 2013).

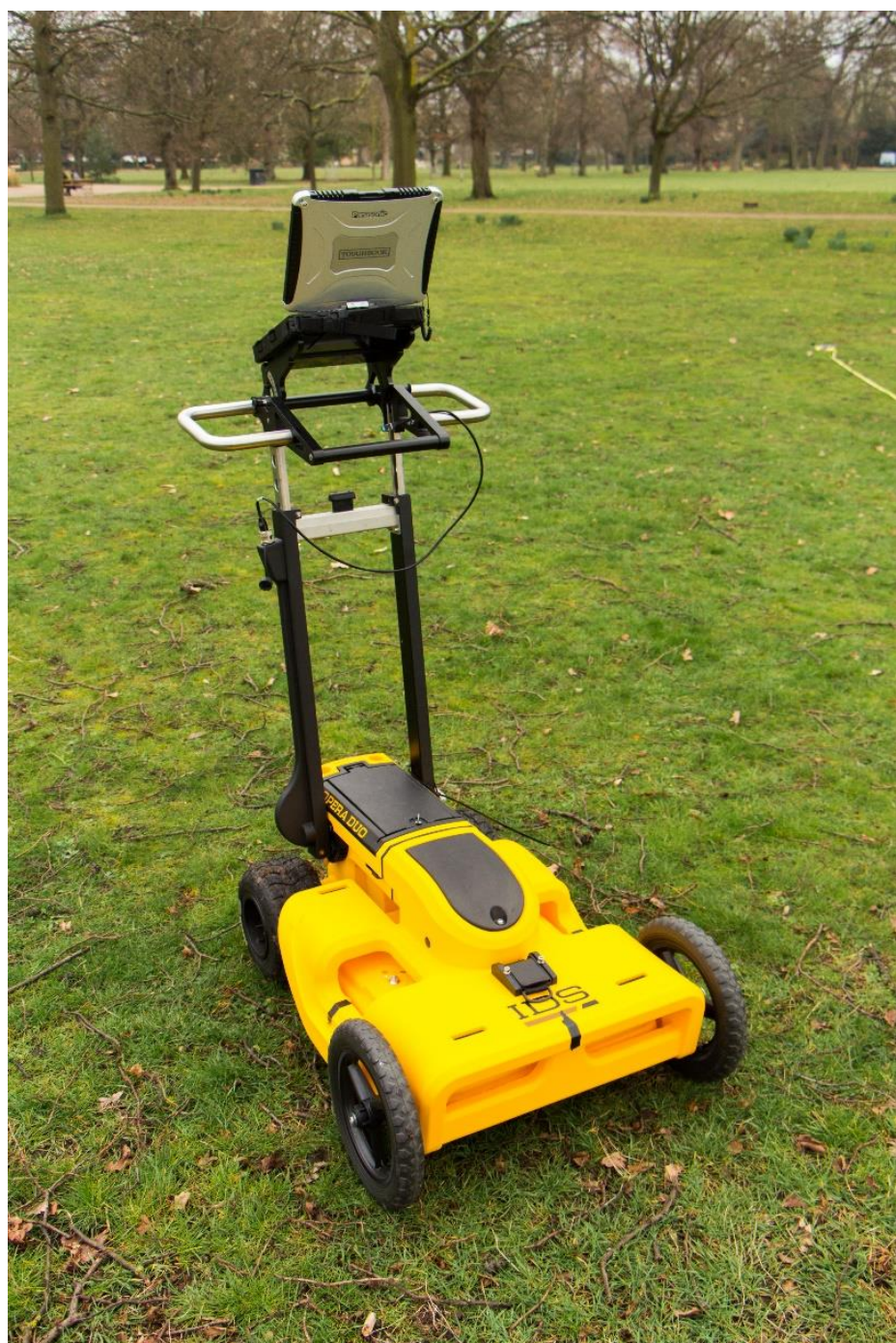


As previously stated, the data processing methodology is divided into three main stages.

841 A pre-processing stage was envisaged, aiming to eliminate clutter-related signal and increase the signal-to-noise ratio (SNR). To this purpose, advanced signal processing techniques were implemented. Moreover, in order to achieve information about the architecture of the entire tree root system, reflections from deeply localised targets were amplified.

\subsubsection{Pre-processing stage}

847 The need for a pre-processing stage arises from the fact that raw GPR data are often

848 corrupted by clutter. This can make the data interpretation difficult, as the response 849 from the real targets can be disguised. In order to ensure the widest possible 850 applicability of the proposed methodology, basic signal processing techniques were 851 considered. Thus, a sequential use of a) zero-offset removal, b) zero correction, c) 852 bandpass filtering and d) time-varying gain was performed.

853 Nevertheless, the application of the aforementioned techniques does not help with the 854 removal of ringing noise, which is a repetitive type of clutter and can appear as horizontal and periodic events. When present, ringing noise can conceal the real target

856 of the investigation, with resulting misinterpretation of results. One of the most 857 effective techniques for ringing noise removal, the Singular Value Decomposition (SVD), 858 was therefore implemented in this stage.

859 The concept behind the SVD filter is that a GPR image can be divided into several sub860 images (eigenimages), each of which contains some of the information relating to the original image. Since components such as ringing noise are highly correlated, it is possible to separate their response from the one given by the real target of the investigation, thus eliminating the clutter to enhance the SNR.

864 Another important advancement in the signal processing stage arises from the need to 865 have information on the real position of the target. As previously stated, the response 866 from a target in a GPR survey is given by a reflection hyperbola, the apex of which 867 corresponds to the position of the buried object. This concept is acceptable for a simple 
location of a target. However, automatic mapping of a tree root system architecture in a 3D domain requires the target to be concentrated in a single point. This will avoid false

870 alarms for root identification. To this effect, a frequency-wavenumber (F-K) migration was applied to GPR data, assuming a constant velocity of the medium and estimating it

872 through an iterative procedure. This allowed to find the permittivity value that best fit 873 the data.

\subsubsection{Tree root tracking algorithm}

875 The implementation of the algorithm for the automatic reconstruction of the tree root system geometry consists of two main parts. In the first part, the main settings, based on fundamental set up hypotheses, are defined (i.e. the outcomes of the previous preprocessing phase, matrix dimensions, and GPR data acquisition settings). In addition, other important variables (i.e. the data acquisition method and the dielectric properties of the medium) are initialised.

881

Subsequently, the pre-processed GPR data undergo an iterative procedure, in order to find a correlation between the amplitude values in different positions of the 3D domain. The steps of the procedure are the following:

- Detection of the target: each amplitude value in the data matrix is compared with a predefined threshold value, in order to identify the reflections that are more likely to belong to tree roots.

- Correlation analysis: a spatial correlation analysis is carried out between the identified reflections.

- Root tracking: where a correlation is found, targets are assembled into vectors which represent the spatial coordinates of the identified root.

- Reconstruction of root system architecture in the 3-D domain: all the vectors are positioned in a 3D environment, based on the previously identified coordinates, to recreate a rendering of the tree root system. 


\subsubsection{Root density evaluation}

896 In this final step, root density is evaluated based on the position and length of the roots 897 obtained in the previous phase. Through the application of a polynomial fitting function, 898 the roots' path was better approximated in a continuous domain, thus allowing for the 899 estimation of the length of each root. Based on this, the volume in which the tree root 900 system resides was divided into reference volumes, and the length of the roots enclosed 901 in each volume was evaluated as follows:

$$
d=\frac{\sum_{i=1}^{n} L_{i}}{V}
$$

902 where $d$ is the density $\left[\mathrm{m} / \mathrm{m}^{3}\right], n$ is the number of roots contained in a reference unit of 903 volume $\left[\mathrm{m}^{3}\right]$ and $L_{i}$ is the length of the root $[\mathrm{m}]$.

\subsubsection{Results and discussion}

905 The advances made here to the GPR data pre-processing phase have allowed a more 906 effective identification of the tree roots, significantly reducing the margin of error. In 907 fact, they made it possible to remove horizontal layers and repeated reflections given 908 by ringing noise through the application of the SVD filter. Figure 20 shows an example 909 of B-scan before (a) and after (b) the application of the SVD filter, from the analysis of 910 which it is clear that the effect of noise-related features is considerably mitigated. 


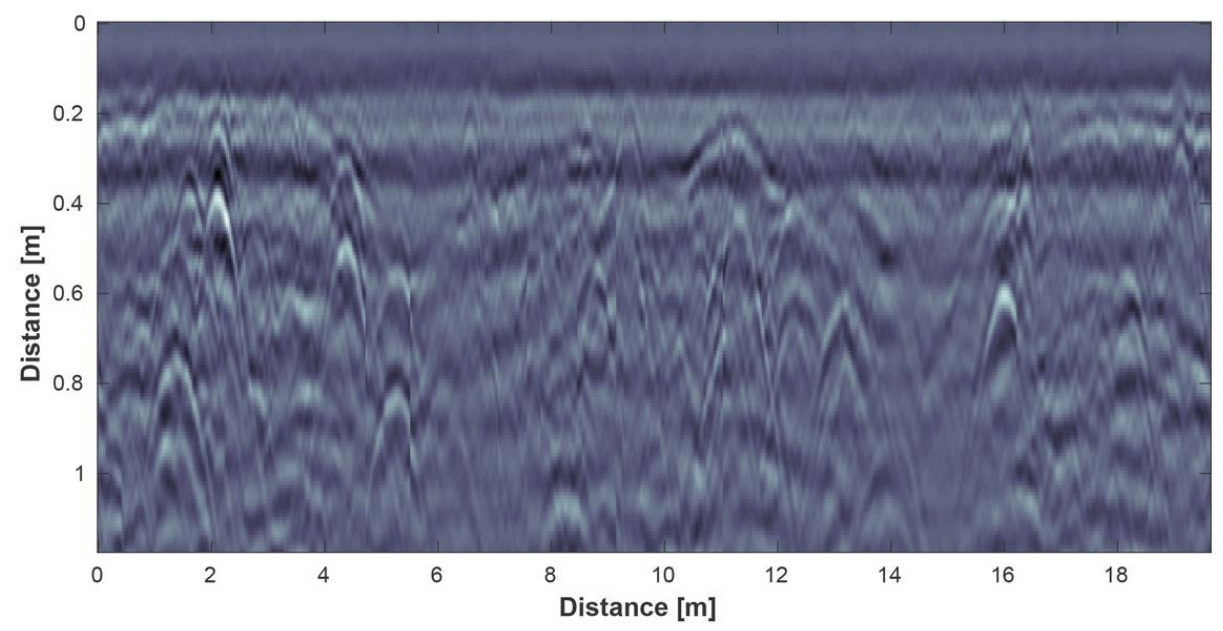

(a)

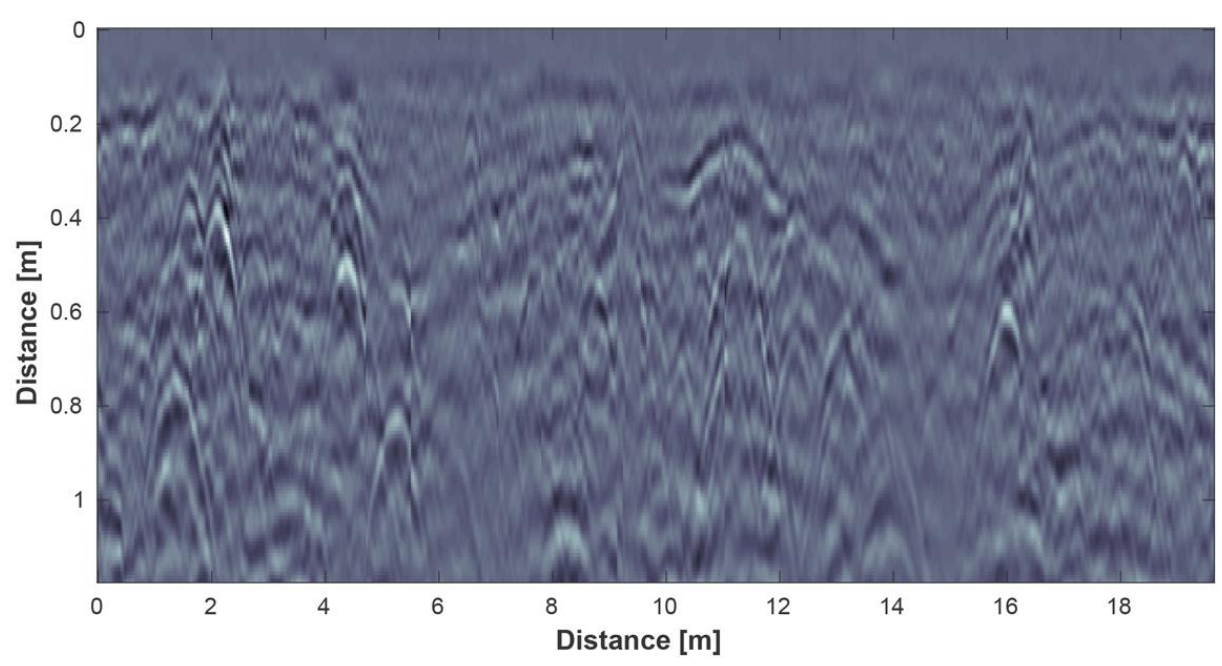

(b)

913 Moreover, the application of F-K migration significantly improved the effectiveness of

914 the subsequent phases of the algorithm, as the margin of error in identifying the true

915 position of the roots was significantly reduced. In fact, the tails of the hyperbole made 916 accurate target detection difficult, as not infrequently points far from the apices (i.e. the

917 real location of the target) were higher than the set threshold. Thus, the migration

918 process increased the reliability of the subsequent steps. Figure 21 shows a comparison

919 between a B-scan before (a) and after (b) the application of the F-K migration. It is 920 evident how the hyperbolic response of the targets has become a single focused point, 921 which corresponds to the target's real position. 


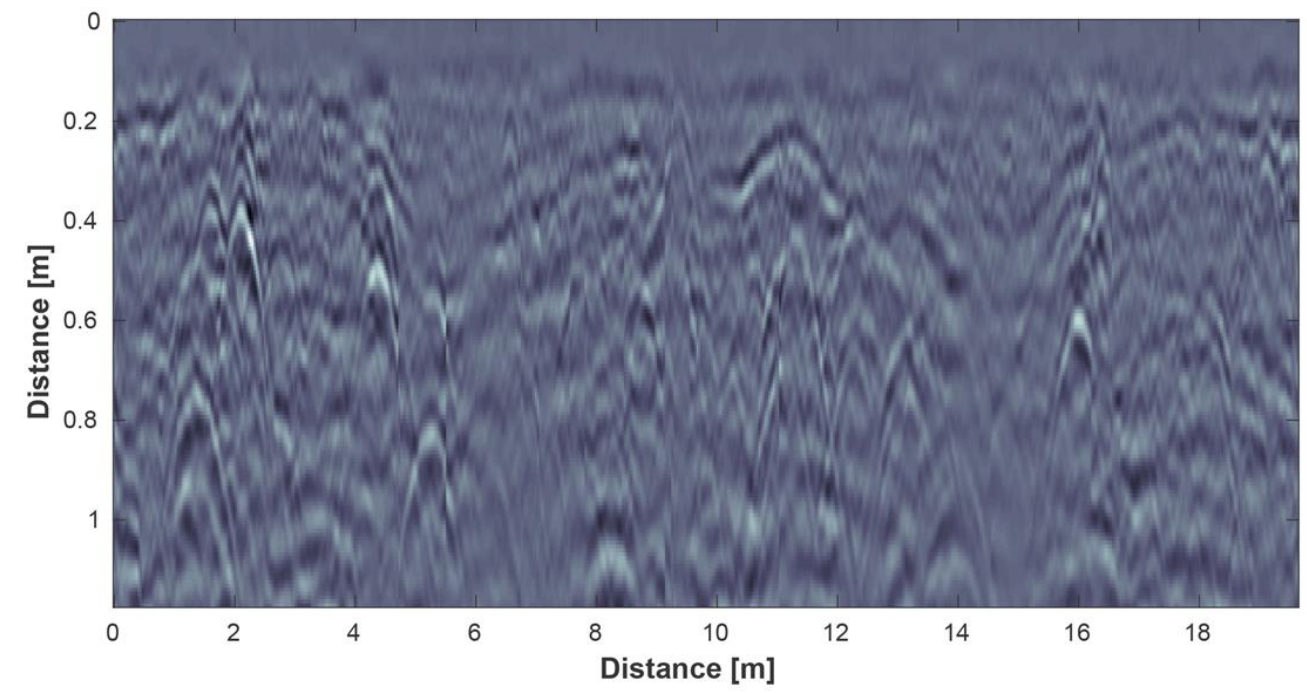

(a)

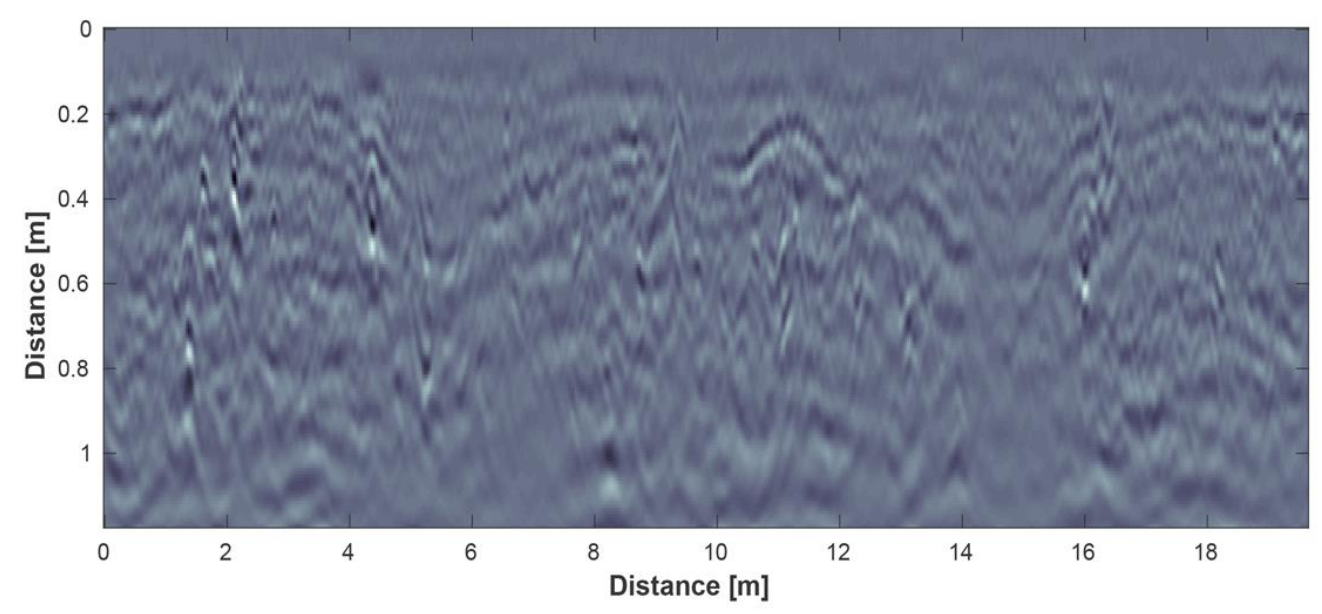

(b)

922

Figure 21: Bscan before (a) and after (b) the application of F-K migration

924 Subsequently, the application of the root tracking algorithm to the processed data 925 allowed for the reconstruction of the tree root system architecture in a three926 dimensional environment. Figure 22 shows the result of this procedure in a 2D planar 927 view (a) and in a 3D environment (b). To make interpreting the results easier, shallow928 buried roots (i.e. within the first $25 \mathrm{~cm}$ of soil) have been represented with a different 929 colour than deeper roots. 


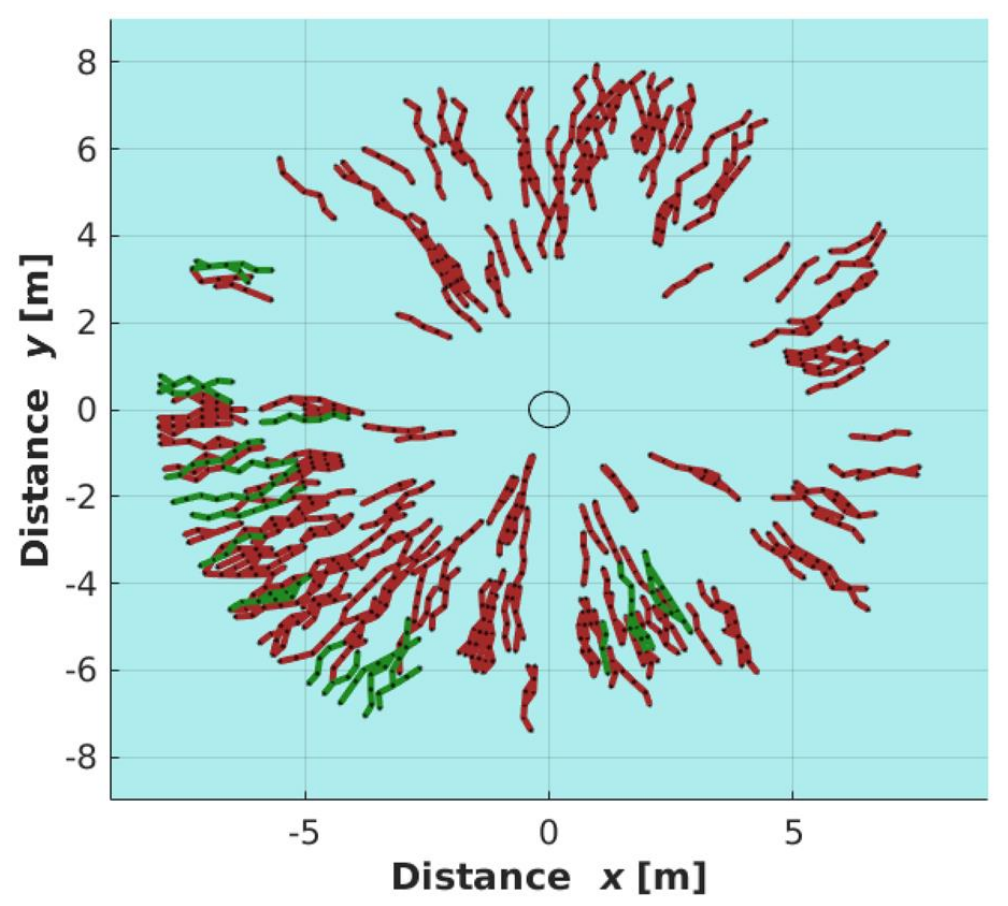

(a)

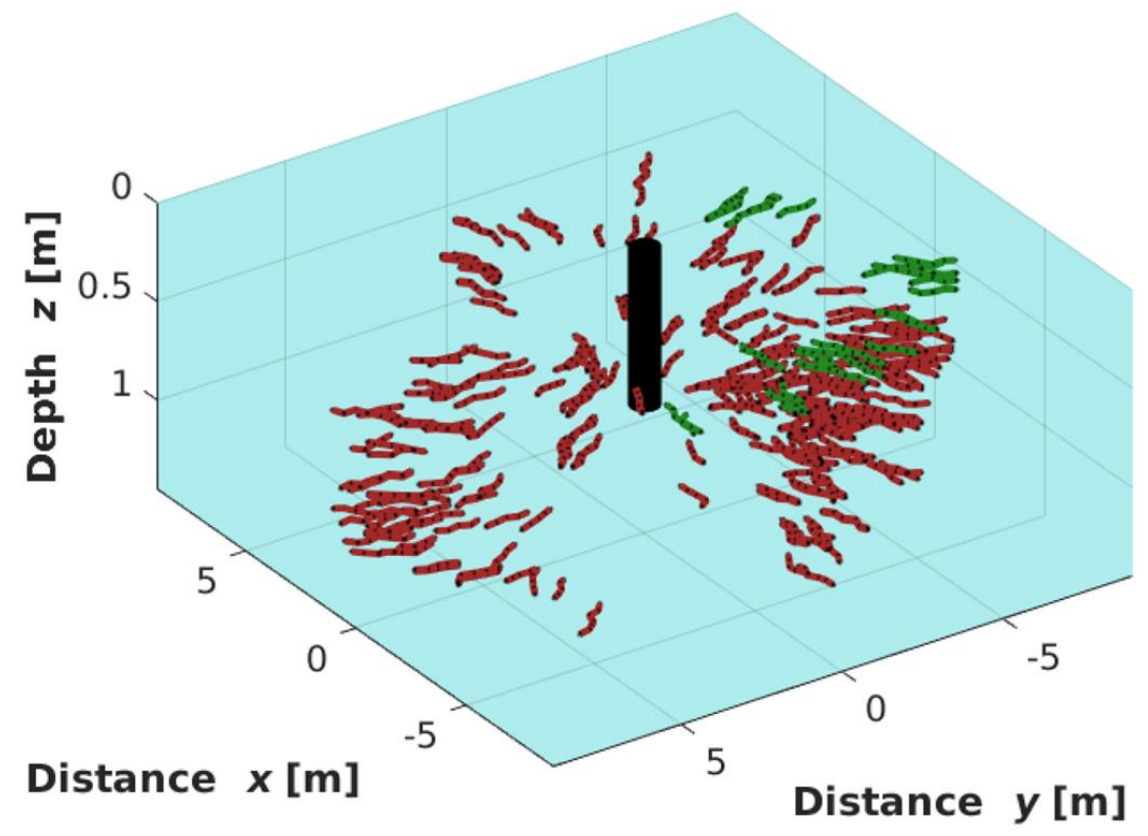

(b) 
932 Results have proven the potential of the algorithm in identifying consistent root paths.

933 Points belonging to the roots were successfully identified and linked together, based on

934 a spatial correlation analysis.

935 From the analysis of B-scans, the strongest reflections resulted to be located within the 936 first $80 \mathrm{~cm}$ of soil. Nevertheless, the application of the time-varying gain function 937 allowed the detection of deeper targets, up to a maximum depth of $1.20 \mathrm{~m}$. This result 938 is in line with what was expected, as generally tree root systems develop in the first $2 \mathrm{~m}$ 939 of subsoil, with the $90 \%$ to the $99 \%$ of roots occurring in the first meter (Crow, 2005).

940 As depicted in Figure 22, root discontinuity is visible in certain areas. Possible 941 explanations for this could be:

- Presence of a higher moisture content (Ortuani, et al., 2013) or a high concentration of clay in certain areas of subsoil (Patriarca, et al. 2013; Tosti, et al., 2016).

- Propagation of tree roots vertically downwards within the soil matrix

946 Furthermore, in order to avoid the inclusion of non-root targets within the soil (cobles 947 and utility futures), the algorithm is programmed to discard shorter roots.

948 The architecture of the root system was then further investigated through the 949 evaluation of root density at different depths, using the proposed equation (Equation 950 6). The domain investigated was divided into reference volumes of $0.3 \mathrm{~m} \times 0.3 \mathrm{~m} \times 0.1$ $951 \mathrm{~m}$ and thus analysed to determine the total root length per reference unit. Figure 23 952 presents the outcomes of this data processing stage. Several areas with a high density 953 of roots can be identified, as shown in Table 3. 


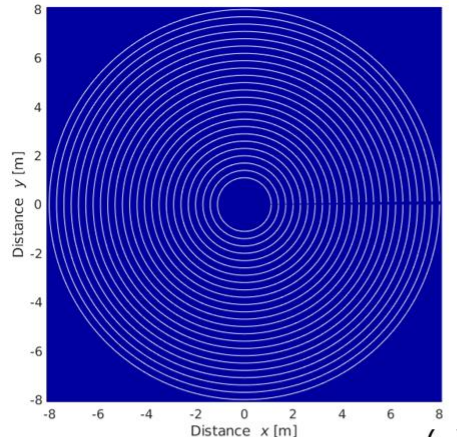

(a)

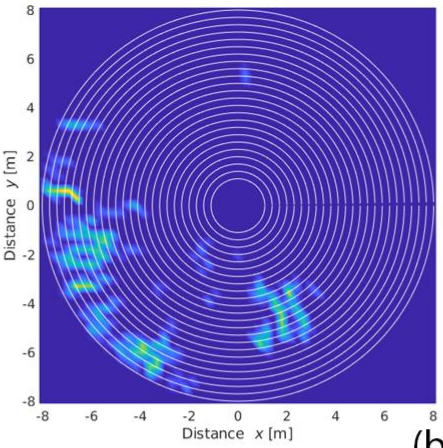

(b)
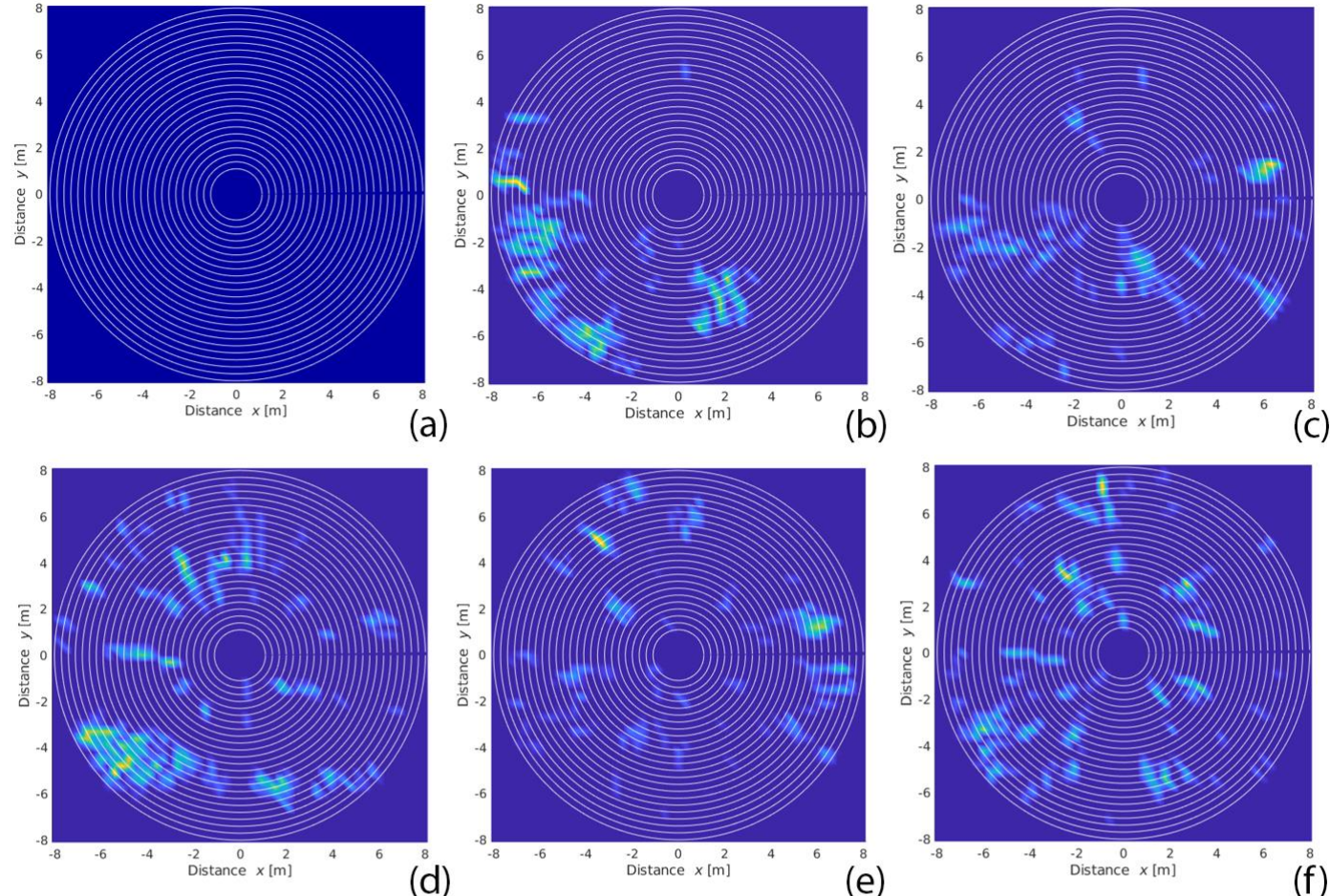

(e)
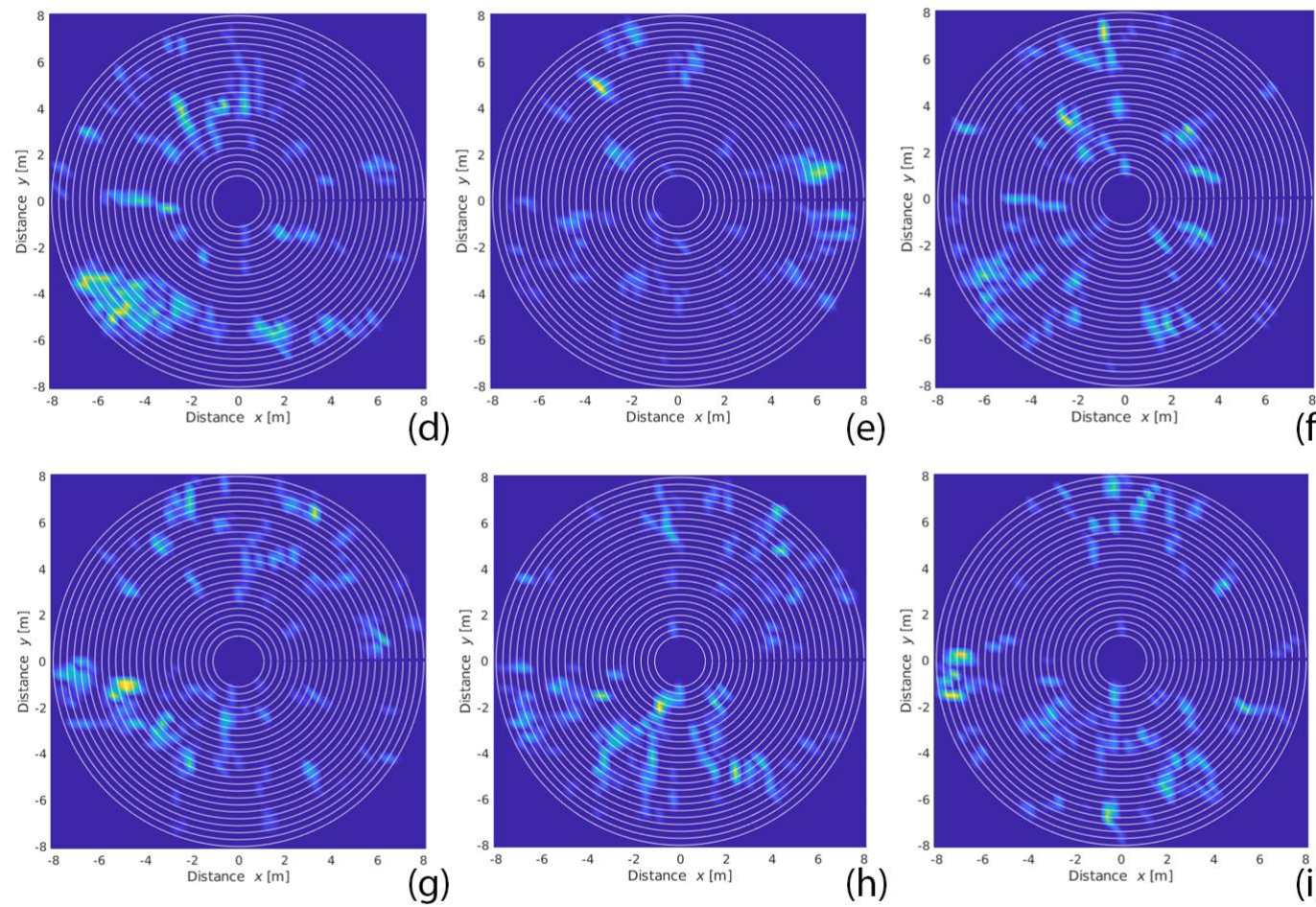

(h)
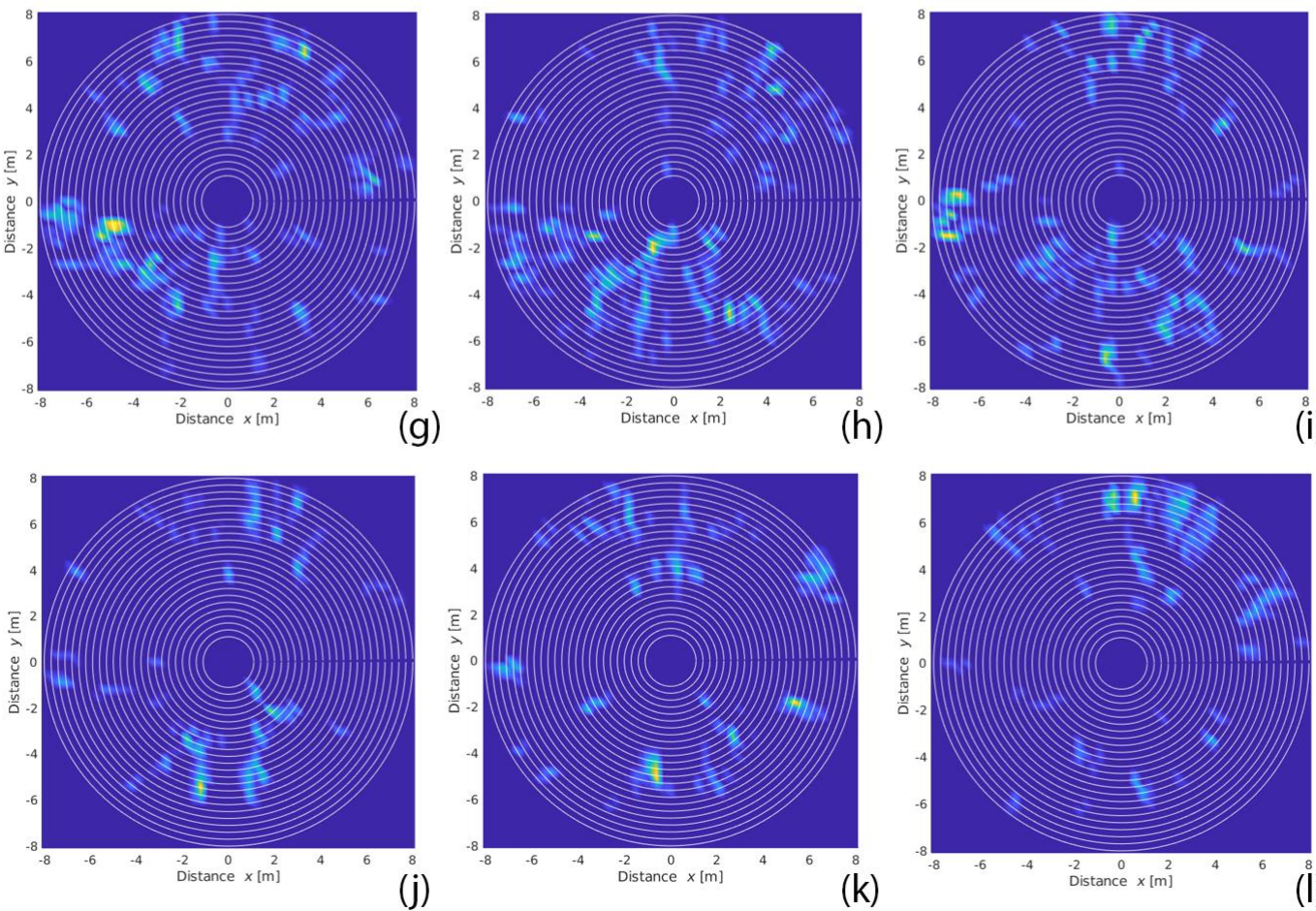

(k)

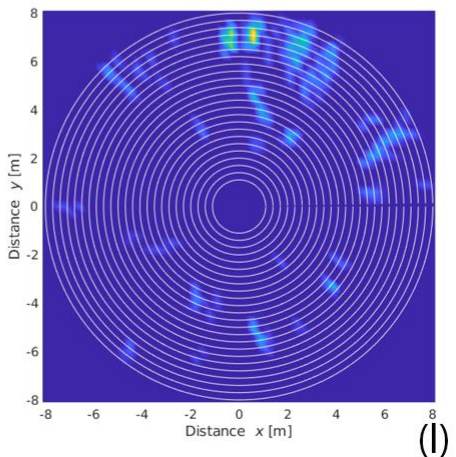

Figure 23: GPR-derived root density maps, related to the following depths: a) from $0 \mathrm{~m}$ to $0.10 \mathrm{~m}$; b) from $0.10 \mathrm{~m}$ to $0.20 \mathrm{~m}$; c) from $0.20 \mathrm{~m}$ to $0.30 \mathrm{~m}$; d) from $0.30 \mathrm{~m}$ to $0.40 \mathrm{~m}$; e) from $0.40 \mathrm{~m}$ to $0.50 \mathrm{~m}$; f) from $0.50 \mathrm{~m}$ to $0.60 \mathrm{~m}$; g) from $0.60 \mathrm{~m}$ to $0.70 \mathrm{~m}$; h) from $0.70 \mathrm{~m}$ to $0.80 \mathrm{~m}$; i) from $0.80 \mathrm{~m}$ to $0.90 \mathrm{~m}$; j) from $0.90 \mathrm{~m}$ to $1.00 \mathrm{~m}$; $k$ ) from $1.00 \mathrm{~m}$ to $1.10 \mathrm{~m}$; l) from $1.10 \mathrm{~m}$ to $1.20 \mathrm{~m}$; 


\begin{tabular}{|c|c|c|c|c|c|}
\hline \multicolumn{6}{|c|}{ Zones of increased density } \\
\hline \multirow{2}{*}{$\begin{array}{l}\text { Depth } \\
\text { [m] }\end{array}$} & \multicolumn{2}{|c|}{$\mathbf{x}$} & \multicolumn{2}{|c|}{ y } & \multirow{2}{*}{$\begin{array}{l}\text { Maximum values } \\
{\left[\mathrm{m} / \mathrm{m}^{3}\right]}\end{array}$} \\
\hline & From [m] & To [m] & From [m] & To [m] & \\
\hline \multirow{3}{*}{$0.10-0.20$} & -6.30 & -7.20 & 0.00 & 0.60 & 1.25 \\
\hline & -6.30 & -6.60 & -3.30 & -3.60 & 1.08 \\
\hline & 2.10 & 2.40 & -3.60 & -3.90 & 1.03 \\
\hline \multirow{6}{*}{$0.20-0.30$} & 5.70 & 6.90 & 0.90 & 1.50 & 2.05 \\
\hline & 0.60 & 1.20 & -2.40 & -3.00 & 1.14 \\
\hline & 6.30 & 6.60 & -4.20 & -4.50 & 1.11 \\
\hline & -6.30 & -6.60 & -1.20 & -1.50 & 1.11 \\
\hline & -4.20 & -4.50 & -2.10 & -2.40 & 1.11 \\
\hline & 0.00 & 0.30 & -3.60 & -3.90 & 1.05 \\
\hline \multirow{6}{*}{$0.30-0.40$} & -2.40 & -6.90 & -3.30 & -5.70 & 1.85 \\
\hline & -0.60 & 0.60 & 3.90 & 4.20 & 1.55 \\
\hline & -1.80 & -2.40 & 3.00 & 3.90 & 1.49 \\
\hline & -2.70 & -4.20 & 0.00 & -0.60 & 1.48 \\
\hline & 0.90 & 2.10 & -5.40 & -6.00 & 1.28 \\
\hline & -6.30 & -6.60 & 2.70 & 3.00 & 1.11 \\
\hline \multirow{5}{*}{$0.40-0.50$} & -3.00 & -3.60 & 4.50 & 5.10 & 2.49 \\
\hline & 5.40 & 6.90 & 0.60 & 1.80 & 1.84 \\
\hline & 6.90 & 7.50 & -0.60 & -1.80 & 1.50 \\
\hline & -1.50 & -1.80 & 6.60 & 6.90 & 1.20 \\
\hline & -2.40 & -2.70 & 1.80 & 2.10 & 1.09 \\
\hline \multirow{11}{*}{$0.50-0.60$} & -0.60 & -0.90 & 6.60 & 7.50 & 1.88 \\
\hline & -2.10 & -2.70 & 3.00 & 3.60 & 1.79 \\
\hline & 2.40 & 3.00 & 2.40 & 3.00 & 1.67 \\
\hline & -5.70 & -6.00 & -3.30 & -4.50 & 1.48 \\
\hline & 1.80 & 2.10 & -5.40 & -5.70 & 1.33 \\
\hline & 3.30 & 3.60 & -1.50 & -1.80 & 1.33 \\
\hline & 3.00 & 3.30 & 0.90 & 1.20 & 1.32 \\
\hline & -1.80 & -2.10 & -3.60 & -3.90 & 1.12 \\
\hline & 1.50 & 1.80 & -1.80 & -2.10 & 1.05 \\
\hline & -3.60 & -3.90 & -3.30 & -3.60 & 1.04 \\
\hline & -6.60 & -6.90 & 2.70 & 3.00 & 1.00 \\
\hline \multirow{10}{*}{$0.60-0.70$} & -4.20 & -5.40 & -0.90 & -1.80 & 2.20 \\
\hline & 3.30 & 3.60 & 6.00 & 6.60 & 1.94 \\
\hline & -2.70 & -4.50 & -2.40 & -3.30 & 1.66 \\
\hline & -1.80 & -2.10 & -4.20 & -4.80 & 1.53 \\
\hline & -1.80 & -2.10 & 6.30 & 7.20 & 1.48 \\
\hline & 6.30 & 6.60 & 0.60 & 0.90 & 1.46 \\
\hline & -3.00 & -3.60 & 4.50 & 5.10 & 1.26 \\
\hline & -6.60 & -7.50 & 0.00 & -0.90 & 1.17 \\
\hline & -0.30 & -0.60 & -2.40 & -3.00 & 1.15 \\
\hline & -1.80 & -2.10 & 3.30 & 3.60 & 1.04 \\
\hline $0.70-0.80$ & -0.30 & -3.60 & -1.50 & -5.10 & 2.25 \\
\hline
\end{tabular}




\begin{tabular}{|c|c|c|c|c|c|}
\hline & 2.40 & 2.70 & -4.50 & -5.40 & 1.94 \\
\hline & 1.50 & 1.80 & -1.80 & -2.10 & 1.60 \\
\hline & 4.20 & 4.80 & 4.50 & 4.80 & 1.55 \\
\hline & 4.20 & 4.50 & 6.00 & 6.60 & 1.49 \\
\hline & 1.50 & 1.80 & -4.80 & -5.10 & 1.33 \\
\hline & 3.60 & 3.90 & -4.50 & -4.80 & 1.33 \\
\hline & -5.10 & -5.40 & -1.20 & -1.50 & 1.29 \\
\hline & 0.00 & -0.60 & 5.10 & 6.00 & 1.18 \\
\hline & 4.80 & 5.10 & 2.40 & 2.70 & 1.06 \\
\hline & -6.60 & -6.90 & -0.30 & -0.60 & 1.05 \\
\hline & -6.30 & -6.90 & -2.40 & -3.60 & 1.04 \\
\hline \multirow{8}{*}{$0.80-0.90$} & -6.60 & -7.50 & 0.30 & -1.80 & 1.74 \\
\hline & -0.30 & -0.60 & -6.60 & -7.20 & 1.36 \\
\hline & 5.40 & 5.70 & -2.10 & -2.40 & 1.27 \\
\hline & 0.90 & 1.50 & 6.60 & 7.20 & 1.21 \\
\hline & 4.50 & 4.80 & 3.00 & 3.30 & 1.16 \\
\hline & 0.00 & -0.30 & 7.20 & 7.50 & 1.14 \\
\hline & 1.80 & 2.10 & -5.40 & -5.70 & 1.10 \\
\hline & 0.00 & -0.30 & -1.50 & -1.80 & 1.03 \\
\hline \multirow{5}{*}{$0.90-1.00$} & -0.90 & -1.80 & -3.30 & -6.00 & 2.22 \\
\hline & 1.20 & 2.10 & -1.20 & -5.40 & 1.65 \\
\hline & 3.00 & 3.30 & 6.60 & 6.90 & 1.24 \\
\hline & 2.10 & 2.40 & 5.10 & 5.70 & 1.20 \\
\hline & 3.00 & 3.30 & 3.90 & 4.20 & 1.06 \\
\hline \multirow{10}{*}{$1.00-1.10$} & 5.10 & 6.00 & -1.80 & -2.10 & 2.43 \\
\hline & -0.30 & -0.90 & -4.20 & -5.40 & 2.25 \\
\hline & 2.70 & 3.00 & -3.00 & -3.60 & 1.64 \\
\hline & 0.30 & 0.60 & 3.60 & 4.20 & 1.39 \\
\hline & -3.00 & -3.60 & -1.80 & -2.40 & 1.39 \\
\hline & 6.00 & 6.90 & 3.30 & 3.90 & 1.27 \\
\hline & -1.50 & -1.80 & 5.70 & 6.60 & 1.26 \\
\hline & -6.30 & -7.20 & -0.30 & -0.60 & 1.19 \\
\hline & 1.50 & 1.80 & -1.80 & -2.10 & 1.13 \\
\hline & -1.20 & -1.50 & 2.70 & 3.30 & 1.11 \\
\hline \multirow{5}{*}{$1.10-1.20$} & -0.60 & 1.20 & 6.30 & 7.20 & 2.29 \\
\hline & 2.40 & 2.70 & 5.70 & 6.90 & 1.26 \\
\hline & 0.60 & 0.90 & 4.20 & 4.50 & 1.24 \\
\hline & 3.90 & 4.20 & -3.30 & -3.60 & 1.15 \\
\hline & 2.10 & 2.40 & 2.70 & 3.00 & 1.03 \\
\hline
\end{tabular}

960 From the analysis of the results, it can be noticed that there is a high density of roots in 961 the south-west quadrant, at a depth between $0.10 \mathrm{~m}$ and $1.10 \mathrm{~m}$. This result could be 962 due to the peculiar location of the investigated tree in the park. In fact, the tree is 963 confined to the north by the presence of a pathway, which requires a higher compaction 964 level than the undisturbed soil. Moreover, root development is not limited to the south- 
965 west direction, as there are no other trees which could compete for the exploitation of 966 soil resources. Nevertheless, we can note the presence of areas of high root density in 967 the east direction, between $0.30 \mathrm{~m}$ and $0.50 \mathrm{~m}$ deep and at a great distance from the 968 trunk. This could be due to the close proximity of another tree, which roots are 969 interconnected with the ones of the investigated system. In fact, in that direction root 970 density gradually decreases, to then increase again towards the limit of the surveyed 971 area, bordering the area potentially affected by the roots of the adjacent tree. Such an 972 outcome is in line with the results provided by Lantini, et al. (2018).

973 The evaluation of tree root density in soil has therefore proven to be an effective tool 974 for the assessment of the root system conditions. Variations in time of root density, 975 obtained by repeating GPR tests at appropriate intervals, could help in the assessment 976 of the root system health. In fact, sudden reductions in root density could be due to the 977 occurrence of diseases or fungal attacks. Thus, acknowledging the problem at its early 978 stage could allow the application of appropriate remedial actions, in order to save the 979 tree and prevent infection from spreading to other trees. 


\section{Conclusion}

982 In this review paper, the authors have presented a significant proportion of the existing 983 literature within the subject area of assessment and monitoring of tree roots and their 984 interaction with the soil. To that effect the nature of tree root systems, their architecture 985 and the factors affecting their development have been covered. Emphasis was paid to 986 establishing the reasons behind the increasing importance of assessment and health monitoring of tree roots and their relationship with the health of trees.

An emphasis is given to the major destructive methods for tree root detection and mapping, followed by a section presenting a summary of the main non-destructive testing methods and the research outputs based on their application for tree root system evaluation. The paper also clearly demonstrated that the investigation of tree root systems using non-destructive testing (NDT) methods is effective and is gaining momentum. As the awareness of the importance of the world's natural heritage is growing, hopefully more desperately needed research and development work will be carried out and efforts will be devoted to this vitally important area of endeavour.

996 Due to its ease of use, its non-intrusiveness nature and its relatively low costs, Ground Penetrating Radar (GPR) was found to be one of the most reliable tools for root inspection. Recent research has focused on root detection and three-dimensional mapping of tree root systems architecture and root diameter, and the evaluation of root diameter in complex urban areas. New research is now focusing on tree root and soil interactions, as well as the interconnectivity of tree roots with one another.

1002 Furthermore, it is important to report that the authors are currently engaged with 1003 research involving novel survey methodologies and data acquisition techniques which 1004 in turn have been applied in assessing a variety of tree species. Promising results have 1005 been obtained within the context of tree roots variations as well as the soil 1006 characterisations.

Advancements in GPR signal processing for tree root assessment and mapping are also under development. To that effect, a case study was presented, focusing on the removal 
of noise-related information for an improved automatic recognition and mapping of tree

1010 roots in a 3D environment.

1011 Regarding the assessment of the root mass density, it is important to conclude that, at 1012 the present time, existing assessment methods are unable to provide accurate 1013 estimations. As has been pointed out earlier, the importance of assessing tree root 1014 density is vital for several purposes, ranging from the health of the tree to the safety of 1015 the surrounding environment (including buildings and infrastructure). It was noted that 1016 a definitive approach is difficult to achieve, as the estimation of root density is an 1017 indirect output of the compiled GPR data. Within this framework, the authors have 1018 proposed a new emerging approach, based on the evaluation of a novel root density 1019 index. Root density is evaluated based on the position and length of the roots, as it is 1020 obtained from the modelling phase of the root mapping algorithm. Results have given 1021 encouraging outcomes, showing that a more reliable estimation of tree root density can 1022 be achieved. More research is now under development, in order to demonstrate the 1023 viability of the proposed algorithm. To this extent, tests on several species of trees, using 1024 different antenna systems (frequencies and type) and survey conditions, are under 1025 development.

\section{Acknowledgements}

1027 This paper is dedicated to the memory of Jonathan West; a friend, a colleague, a 1028 forester, a conservationist and an environmentalist, who died following an accident in 1029 the woodland that he loved.

1030 We would like to thank The Lord Faringdon Charitable Trust and The Schroder 1031 Foundation for supporting this project. 


\section{References}

1033 al Hagrey, S. A., 2007. Geophysical imaging of root-zone, trunk, and moisture 1034 heterogeneity. Journal of Experimental Botany, Volume 58, pp. 839-854.

1035

Alani, A. M., Aboutalebi, M. \& Kilic, G., 2013. Applications of ground penetrating radar 1036 (GPR) in bridge deck monitoring and assessment. Journal of Applied Geophysics, Volume 1037 97, pp. 45-54.

1038

Alani, A. M. et al., 2018. Mapping the root system of matured trees using ground 1039 penetrating radar. s.I., IEEE, pp. 1-6.

1040 Alani, A. M. \& Tosti, F., 2018. GPR applications in structural detailing of a major tunnel 1041 using different frequency antenna systems. Construction and Building Materials, 1042 Volume 158, pp. 1111-1122.

1043 Amato, A. et al., 2009. Multi - electrode 3D resistivity imaging of alfalfa root zone. 1044 European Journal of Agronomy, Volume 31, pp. 213-222.

1045 Amato, M. et al., 2008. In situ detection of tree root distribution and biomass by multi 1046 electrode resistivity imaging. Tree Physiology, Volume 28, pp. 1441-1448.

1047 Amato, M. \& Ritchie, J. T., 2002. Spatial Distribution of Roots and Water Uptake of Maize 1048 (Zea mays L.) as Affected by Soil Structure. Crop Science, Volume 42, pp. 773-780.

1049 Anagnostakis, S. L., 1987. Chestnut blight: the classical problem of an introduced 1050 pathogen. Mycologia, 79(1), pp. 23-37.

1051 Aukema, J. E. et al., 2011. Economic impacts of non-native forest insects in the 1052 continental United States. PLoS One, 6(9).

1053 Aulen, M. \& Shipley, B., 2012. Non-destructive estimation of root mass using electrical 1054 capacitance on ten herbaceous species. Plant and Soil, 355(1-2), pp. 41-49.

1055 Barker, P. A., 1983. Some urban trees of California: manteinance problems and genetic 1056 improvement possibilities. s.l., s.n., pp. 47-54. 
1057

1058

1059

1060

1061

1062

1063

1064

1065

1066

1067

1068

1069

1070

1071

1072

1073

1074

1075

1076

1077

1078

1079

1080

1081

Barton, C. V. M. \& Montagu, K. D., 2004. Detection of tree roots and determination of root diameters by ground penetrating radar under optimal conditions. Tree Physiology, Volume 24, pp. 1323-1331.

Basso, B. et al., 2010. Two-Dimensional Spatial and Temporal Variation of Soil Physical Properties in Tillage Systems Using Electrical Resistivity Tomography. Agronomy Journal, Volume 102.

Bassuk, N., Grabosky, J., Mucciardi, A. \& Raffel, G., 2011. Ground-penetrating Radar Accurately Locates Tree Roots in Two Soil Media Under Pavement. Arboriculture \& Urban Forestry, Volume 37, pp. 160-166.

Benedetto, A. et al., 2017. Railway ballast condition assessment using groundpenetrating radar - An experimental, numerical simulation and modelling development. Construction and Building Material, Volume 140, pp. 508-520.

Benedetto, A., Tosti, F., Bianchini Ciampoli, L. \& D'Amico, F., 2017. An overview of ground-penetrating radar signal processing techniques for road inspections. Signal Processing, Volume 132, pp. 201-209.

Benedetto, A. et al., 2013. Soil moisture mapping using GPR for pavement applications. s.I., IWAGPR 2013 - Proceedings of the 2013 7th International Workshop on Advanced Ground Penetrating Radar.

Benedetto, A. et al., 2015. Mapping the spatial variation of soil moisture at the large scale using GPR for pavement applications. Near Surface Geophysics, 13(3), pp. 269-278.

Benedetto, A., Tosti, F., Schettini, G. \& Twizere, C., 2011. Evaluation of geotechnical stability of road using GPR. s.I., 2011 6th International Workshop on Advanced Ground Penetrating Radar (IWAGPR 2011).

Besson, A. et al., 2004. Structural heterogeneity of the soil tilled layer as characterized by 2D electrical resistivity surveying. Soil \& Tillage Research, Volume 79, pp. 239-249. 

of GPR Data for Road Surveys. Geosciences, 9(96).

1084

1085

1086

1087

1088

1089

1090

1091

1092

1093

1094

1095

1096

1097

1098

1099

1100

1101

1102

1103

1104

1105

Biddle, G., 2001. Tree root damage to buildings. In: C. Vipulanandan, M. B. Addison \& M. Hasen, eds. Expansive Clay Soils and Vegetative Influence on Shallow Foundations. s.l.:s.n., pp. 1-23.

Birouste, M. et al., 2014. Measurement of fine root tissue density: a comparison of three methods reveals the potential of root dry matter content. Plant and Soil, 374(1-2), pp. 299-313.

Blunt, S. M., 2008. Trees and pavements - are they compatible?. Arboricultural Journal, Volume 31, pp. 73-80.

Bohm, W., 2012. Methods of studying root systems. s.l.:Springer Science \& Business Media.

Bonan, G. B., 1992. Soil temperature as an ecological factor in boreal for ests. In: H. H. Shugart, R. Leemans \& G. B. Bonan, eds. A Systems Analysis of the Global Boreal Forest. s.l.:Cambridge University Press, pp. 126-143.

Borden, K. A. et al., 2014. Estimating coarse root biomass with ground penetrating radar in a tree-based intercropping system. Agroforestry Systems, 88(4), pp. 657-669.

Boyer, J. S., 1995. Roots and root systems. In: Water relations of plants and soils. s.I.:Academic Press, Inc..

Brennan, G., Patch, D. \& Stevens, F. R. W., 1997. Tree Roots and Underground Pipes Arboriculture Research Note, s.l.: Arboricultural Advisory \& Information Service.

Brudi, E. \& Wassenaer, P., 2002. Trees and Statics: Non-Destructive Failure Analysis. Champaign, Illinois, International Society of Arboriculture, pp. 53-70.

Bucur, V., 2006. Acoustics of wood. s.l.:Springer Science \& Business Media. 
1106 Butnor, J. R. et al., 2003. Utility of Ground-Penetrating Radar as a Root Biomass Survey

1107 Tool in Forest Systems. Soil Science Society of America Journal, Volume 67, pp. 160711081615.

1109 Butnor, J. R. et al., 2001. Use of ground-penetrating radar to study tree roots in the 1110 southeastern United States. Tree Physiology, Volume 21, pp. 1269-1278.

1111 Butnor, J. R. et al., 2008. Using Ground-Penetrating Radar to Estimate Tree Root Mass 1112 Comparing Results from Two Florida Surveys. In: B. J. Allred, J. J. Daniels \& M. R. Ehsami, 1113 eds. Handbook of Agricultural Geophysics. Boca Raton: CRC Press, pp. 375-382.

1114 Buza, A. K. \& Divos, F., 2016. Root stability evaluation with non - destructive techniques. 1115 Acta Silvatica et Lignaria Hungarica, Volume 12, pp. 125-134.

1116 Buza, A. K. \& Goncz, B., 2015. Comparison of trees and NDT methods. Wood research, 1117 Volume 60, pp. 45-58.

1118 Canadian Forest Service, 2015. Trees, insects and diseases of Canada's forests - Natural Resources Canada.

[Online]

1120 Available at: https://tidcf.nrcan.gc.ca/en/diseases/factsheet/16

1121 Caneva, G., Ceschin, S. \& De Marco, G., 2006. Mapping the risk of damage from tree

1122 roots for the conservation of archaeological sites: the case of the Domus Aurea, Rome.

1123 Conservation and Management of Archaeological Sites, pp. 163-170.

1124 Caneva, G., Galotta, G., Cancellieri, L. \& Savo, V., 2009. Tree roots and damages in the 1125 Jewish catacombs of Villa Torlonia (Roma). Journal of Cultural Heritage, pp. 53-62.

1126 Cermak, J., Hruska, J., Martinkova, M. \& Prax, A., 2000. Urban tree root systems and 1127 their survival near houses analyzed using ground penetrating radar and sap flow 1128 techniques. Plant and Soil, Volume 219, pp. 103-116.

1129 Conyers, L. B., 2002. Ground penetrating radar. Encyclopedia of Imaging Science and 1130 Technology. 
1131 Costello, L. R. \& Jones, K. S., 2003. Reducing infrastructure damage by tree roots: a 1132 compendium of strategies. s.l.:Western Chapter of the International Society of 1133 Arboriculture (WCISA).

1134 Coutts, M. P., 1983. Root architecture and tree stability. Plant and Soil, pp. 171-188.

1135 Crow, P., 2005. The influence of soils and species on tree root depth. Edinburgh: Forestry 1136 commission.

1137 Cui, X. H. et al., 2011. Modeling tree root diameter and biomass by ground-penetrating radar. Science China Earth Sciences, 54(5), pp. 711-719.

1139 Cutler, D. \& Richardson, I. B. K., 1981. Tree roots and buildings. Construction Press.

1140 Daniels, D. J., 1996. Surface - penetrating radar. Electronics \& Communication 1141 Engineering Journal, pp. 165-182.

1142 Daniels, D. J., 2004. Ground Penetrating Radar. s.I.:let.

1143 Day, R. W., 1991. Damage of structures due to tree roots. Journal of Performance of 1144 Constructed Facilities, pp. 200-207.

1145 Divos, F., Bejo, L. \& Toth, A., 2009. Instrument supported tree evaluation in Hungary. 1146 Beijing, Beijing Forestry University, pp. 71-76.

1147 Driscoll, R., 1983. The influence of vegetation on the swelling and shrinking of clay soils 1148 in Britain. Geotechnique, pp. 93-105.

1149 Eshel, A. \& Beeckman, T., 2013. Plant roots: the hidden half. 3rd ed. ed. New York: Marcel 1150 Dekker.

1151 Fay, N., 2014. Appraisal of Trees at Walpole Park, London: s.n.

1152 Francis, J. K., Parresol, B. R. \& de Patino, J. M., 1996. Probability of Damage to Sidewalks 1153 and Curbs by Street Trees in the Tropics. Journal of Arborculture, Volume 22. 
1154 Gibbs, J. N., 1978. Intercontinental epidemiology of Dutch elm disease. Annual Review 1155 of Phytopathology, 16(1), pp. 287-307.

1156 Goodman, D., 1994. Ground - penetrating radar simulation in engineering and 1157 archaeology. Geophysics, Volume 2, pp. 224-232.

1158 Grabianowski, M., Manley, B. \& Walker, J. C. F., 2006. Acoustic measurements on 1159 standing trees, logs and green lumber. Wood Science and Technology, 40(3), pp. 2051160216.

1161 Grabosky, J., Bassuk, N., Irwin, L. \& Van Es, H., 1998. Pilot field study of structural soil 1162 materials in pavement profiles. Champaign, IL, International Society of Arboriculture, pp. $1163 \quad 210-221$.

1164 Grabosky, J., Haffner, E. \& Bassuk, N., 2009. Plant available moisture in stone-soil media 1165 for use under pavement while allowing urban tree root growth. Arboriculture \& Urban 1166 Forestry, 35(5), pp. 271-278.

1167 Gregory, P. J., 2006. Plant roots: growth, activity and interaction with soils. s.I.:Blackwell 1168 Publishing.

1169 Gregory, P. J. et al., 2003. Non-invasive imaging of roots with high resolution X-ray micro1170 tomography. In: Roots: the dynamic interface between plants and the Earth. Dordrecht: 1171 Springer, pp. 351-359.

1172 Guo, L. et al., 2013. Application of ground penetrating radar for coarse root detection 1173 and quantification: a review. Plant and Soil, Volume 362, pp. 1-23.

1174 Hansen, E. M. \& Goheen, E. M., 2000. Phellinus weirii and other native root pathogens 1175 as determinants of forest structure and process in western North America. Annual 1176 review of phytopathology, 38(1), pp. 515-539.

1177 Hargreaves, C. E., Gregory, P. J. \& Bengough, A. G., 2009. Measuring root traits in barley 1178 (Hordeum vulgare ssp. vulgare and ssp. spontaneum) seedlings using gel chambers, soil 1179 sacs and X-ray microtomography. Plant and Soil, 316(1-2), pp. 285-297. 
1180

1181

1182

1183

1184

1185

1186

1187

1188

1189

1190

1191

1192

1193

1194

1195

1196

1197

1198

1199

1200

1201

1202

1203

Heeraman, D. A., Hopmans, J. W. \& Clausnitzer, V., 1997. Three dimensional imaging of plant roots in situ with X-ray computed tomography. Plant and soil, 189(2), pp. 167-179.

Heeraman, D. A. \& Juma, N. G., 1993. A comparison of minirhizotron, core and monolith methods for quantifying barley (Hordeum vulgare L.) and fababean (Vicia faba L.) root distribution. Plant and Soil, Volume 148, pp. 29-41.

Henwood, K., 1973. A structural model of forces in buttressed tropical rain forest trees. Biotropica, pp. 83-89.

Hruska, J., Cermak, J. \& Sustek, S., 1999. Mapping tree root systems with groundpenetrating radar. Tree Physiology, Volume 19, pp. 125-130.

Huisman, J., Hubbard, S., Redman, J. \& Annan, A. P., 2003. Measuring soil water content with ground penetrating radar: a review. Vadose Zone Journal, 2(4), pp. 476-491.

Innes, J. L., 1993. Forest health: its assessment and status. s.I.: Cab International.

Iwase, J. et al., 2015. Non-invasive acoustic sensing of belowground wooden tissues: possible applications to spatial mapping of soil usage by tree roots. Environmental Control in Biology, 53(3), pp. 175-179.

Jackson, R. B. et al., 1996. A global analysis of root distributions for terrestrial biomes. Oecologia, pp. 389-411.

Jol, H. M. ed., 2008. Ground penetrating radar theory and applications. s.I.:Elsevier.

Kaestner, A., Schneebeli, M. \& Graf, F., 2006. Visualizing three-dimensional root networks using computed tomography. Geoderma, 136(1-2), pp. 459-469.

Kearey, P., Brooks, M. \& Hill, I., 2013. An introduction to geophysical exploration. s.l.:John Wiley \& Sons.

Kopinga, J., 1994. Aspects of the damage to asphalt road pavings caused by tree roots. Savoy, IL, International Society of Arboriculture, pp. 165-178. 
Krainyukov, A. \& Lyaksa, I., 2016. Detection of tree roots in an urban area with the use

1205 of ground penetrating radar. Transport and Telecommunication, Volume 17, pp. 3621206370.

1207 Lambot, S., Javaux, M., Hupet, F. \& Vanclooster, M., 2002. A global multilevel coordinate search procedure for estimating the unsaturated soil hydraulic properties. Water Resources Research, 38(11).

1210 Lantini, L. et al., 2018. Use of ground penetrating radar for assessing interconnections between root systems of different matured tree species. Cassino, Italy, IEEE.

1212 Leskovar, D. I., Cantliffe, D. J. \& Stoffella, P. J., 1994. Transplant production systems 1213 influence growth and yield of fresh-market tomatoes. Journal of the American Society 1214 for Horticultural Science, 119(4), pp. 662-668.

1215 Liebhold, A. M. et al., 2012. Live plant imports: the major pathway for forest insect and 1216 pathogen invasions of the US. Frontiers in Ecology and the Environment, 10(3), pp. 1351217143.

1218 Lindsey, P. \& Barlow, L., 1994. The Design of Structural Soil Mixes for Trees in Urban 1219 Areas. s.l.:s.n.

1220 Loh, F. C. W., Grabosky, J. \& Bassuk, N., 2003. Growth response of Ficus benjamina to 1221 limited soil volume and soil dilution in a skeletal soil container study. Urban Forestry \& 1222 Urban Greening, Volume 2, pp. 53-62.

1223 Loizos, A. \& Plati, C., 2007. Accuracy of ground penetrating radar horn-antenna 1224 technique for sensing pavement subsurface. IEEE Sensor Journal, 7(5), pp. 842-850.

1225 Loperte, A. et al., 2006. 2D and 3D high resolution geoelectrical tomography for non 1226 destructive determination of the spatial variability of plant root distribution: laboratory experiments and field measurements. Geophysical Research Abstracts, Volume 8.

1228 Lorenzo, H., Pérez - Gracia, V., Novo, A. \& Armesto, J., 2010. Forestry applications of 1229 ground - penetrating radar. Forest Systems, Volume 19, pp. 5-17. 
1230 Lucke, T. et al., 2011. Using permeable pavements to promote street tree health, to

1231 minimize pavement damage and to reduce stormwater flows. Porto Alegre, Brazil, s.n.

1232 Lundström, T., Jonsson, M. J. \& Kalberer, M., 2007. The root-soil system of Norway 1233 spruce subjected to turning moment: resistance as a function of rotation. Plant and Soil, $1234300(1-2)$, pp. 35-49.

1235 MacLeod, R. D. \& Cram, J. W., 1996. Forces exerted by tree roots, s.I.: Arboriculture 1236 Research and Information Note-Department of the Environment (United Kingdom).

1237 Mahesh, M., 2002. The AAPM/RSNA physics tutorial for residents: search for isotropic 1238 resolution in CT from conventional through multiple-row detector. Radiographics, 22(4), 1239 pp. 949-962.

1240 Majdi, H., 1996. Root sampling methods - applications and limitations of the 1241 minirhizotron. Plant and Soli, Volume 185, pp. 255-258.

1242 Majdi, H. et al., 2005. Measuring fine root turnover in forest ecosystems. Plant and Soil, 1243 pp. 1-8.

1244 Marchi, L. et al., 2018. State of the Art on the Use of Trees as Supports and Anchors in 1245 Forest Operations. Forests, 9(8).

1246 McPherson, E. G. \& Peper, P., 2000. Costs due to conflicts between street tree root 1247 growth and hardscape. Cohasset, CA, Western Chapter (International Society of 1248 Arboriculture), pp. 15-18.

1249 Mooney, S. J., Pridmore, T. P., Helliwell, J. \& Bennet, M. J., 2012. Developing X-ray 1250 Computed Tomography to non-invasively image 3-D root systems architecture in soil. 1251 Plant and Soil, Volume 352, pp. 1-22.

1252 Mullaney, J., Lucke, T. \& Trueman, S. J., 2015. A review of benefits and challenges in 1253 growing street trees in paved urban enviroments. Landscape and Urban Planning, 1254 Volume 134, pp. 157-166. 
1255 Neumann, G., George, T. S. \& Plassard, C., 2009. Strategies and methods for studying 1256 the rhizosphere-the plant science toolbox. Plant and Soil, 321(1-2), pp. 431-456.

1257 Nicoll, B. C. \& Armstrong, A., 1998. Development of Prunus root systems in a city street:

1258 Pavement damage and root architecture. Arboricultural Journal, pp. 259-270.

1259 Nobel, P. S., 2003. Environmental biology of agaves and cacti. s.I.:Cambridge University 1260 Press.

1261 Ortuani, B., et al., 2013. A non-invasive approach to monitor variability of soil water content with electromagnetic methods. Procedia Environmental Sciences, Volume 19, pp. 446-455.

1264 Ow, L. F. \& Sim, E. K., 2012. Detection of urban tree roots with the ground penetrating 1265 radar. Plant Biosystems, Volume 146, pp. 288-297.

1266 Pallardy, S. G., 2008. Physiology of Woody Plants. s.l.:Academic Press.

1267 Panissod, C., Michot, D., Benderitter, Y. \& Tabbagh, A., 2001. On the effectiveness of 2D 1268 electrical inversion results: an agricultural case study. Geophysical Prospecting, Volume 1269 49, pp. 570-576.

1270 Patriarca, C., T et al., 2013. Frequency dependent electric properties of homogeneous multi-phase lossy media in the ground-penetrating radar frequency range. Journal of

1272 Applied Geophysics, Volume 97, pp. 81-88.

1273 Perret, J. S., Al-Belushi, M. E. \& Deadman, M., 2007. Non-destructive visualization and 1274 quantification of roots using computed tomography. Soil Biology and Biochemistry, 1275 39(2), pp. 391-399.

1276 Pokorny, J. D. et al., 2003. Urban tree risk management: a community guide to program 1277 design and implementation. s.I.:s.n.

1278 Potin, D., Duflos, E. \& Vanheeghe, P., 2006. Landmines Ground-Penetrating Radar Signal 1279 Enhancement by Digital Filtering. IEEE Transaction on Geoscience and Remote Sensing, 1280 Volume 44, pp. 2393-2406. 
1281 Randrup, T. B., McPherson, E. G. \& Costello, L. R., 2001. A review of tree root conflicts 1282 with sidewalks, curbs, and roads. Urban Ecosystems, Volume 5, pp. 209-225.

1283 Randrup, T. B., McPherson, E. G. \& Costello, L. R., 2001. Tree root intrusion in sewer 1284 systems: review of extent and costs. Journal of Infrastructure System, pp. 26-31.

1285 Richardson, D. M. et al., 2001. Naturalization and invasion of alien plants: concepts and 1286 definitions. Diversity and Distributions, 6(2), pp. 93-107.

1287 Rishbeth, J., 1972. Resistance to fungal pathogens of tree roots. Proceedings of the Royal 1288 Society of London. Series B. Biological Sciences, 181(1064), pp. 333-351.

1289 Roberts, J., Jackson, N. \& Smith, M., 2006. Tree Roots in the Built Environment. s.I.:Centre 1290 for Ecology and Hydrology (Great Britain).

1291 Samouelian, A. et al., 2005. Electrical resistivity survey in soil science: a review. Soil \& 1292 Tillage Research, Volume 83, pp. 173-193.

1293 Samuelson, L. J. et al., 2008. Growth and physiology of loblolly pine in response to long1294 term resource management: defining growth potential in the southern United States. 1295 Canadian Journal of Forest Research, 38(4), pp. 721-732.

1296 Santini, A. et al., 2012. Biogeographical patterns and determinants of invasion by forest 1297 pathogens in Europe. New Phytologist.

1298 Satriani, A., Loperte, A., Proto, M. \& Bavusi, M., 2010. Building damage caused by tree 1299 roots: laboratory experiments of GPR and ERT surveys. Advances in Geosciences, pp. $1300 \quad 133-137$.

1301 Schrock, B. J., 1994. Existing sewer evaluation and rehabilitation. s.I.:s.n.

1302 Schuurman, J. J. \& Goedewaagen, M. A. J., 1965. Methods for the examination of root 1303 systems and roots, s.l.: Wageningen: Centre for agricultural publications and 1304 documentation. 

fir and red alder seedlings. Ecology, pp. 30-45.

1307 Smit, A. L. et al., 2013. Root Methods: A Handbook. s.I.:Springer Science \& Business 1308 Media.

1309 Stoke, A., 1994. Response of young trees to wind: effects on root architecture and 1310 anchorage strength, York: s.n.

1311 Stokes, A. et al., 2002. An evaluation of different methods to investigate root system architecture of urban trees in situ: I. Ground-penetrating radar. Journal of Arboriculture, pp. 2-10.

1314 Stone, E. L. \& Kalisz, P. J., 1991. On the maximum extent of tree roots. Forest Ecology and Management, pp. 59-102.

1316 Stover, D. B., Day, F. P., Butnor, J. R. \& Drake, B. G., 2007. Effect of elevated CO2 on 1317 coarse-root biomass in Florida scrub detected by ground-penetrating radar. Ecology, 1318 88(5).

1319 Strong, W. L. \& La Roi, G. H., 1983. Root-system morphology of common boreal forest trees in Alberta, Canada. Canadian Journal of Forest Research, pp. 1164-1173.

1321 Sullivan, R. H., Gemmell, R. S., Schafer, L. A. \& Hurst, W. D., 1977. Economic analysis, 1322 root control, and backwater flow control as related to infiltration/inflow control. 1323 Cincinnati: s.n.

1324 Sustek, S., Hruska, J., M., D. \& Michalek, T., 1999. Root surfaces in the large oak tree estimated by image analysis of the map obtained by the ground penetrating radar. Journal of Forest Science, Volume 45, pp. 139-143.

1327 Taylor, H. M., Upchurch, D. R., Brown, J. M. \& Rogers, H. H., 1991. Some methods of root 1328 investigations. In: Developments in Agricultural and Managed Forest Ecology. 1329 s.l.:Elsevier, pp. 553-564. 
1330 Tosti, F., Bianchini Ciampoli, L., Brancadoro, M. G. \& Alani, A. M., 2018a. GPR 1331 applications in mapping the subsurface root system of street trees with road safety1332 critical implications. Advances in transportation studies, Volume 44.

1333 Tosti, F. et al., 2018b. An experimental-based model for the assessment of the mechanical properties of road pavements using ground-penetrating radar. Construction and Building Materials, Volume 165, pp. 966-974.

Tosti, F. et al., 2016. GPR analysis of clayey soil behaviour in unsaturated conditions for pavement engineering and geoscience applications. Near Surface Geophysics, Volume 14 (2), pp. 127-144.

1339 Tosti, F. \& Pajewski, L., 2015. Applications of Radar Systems in Planetary Sciences: An 1340 Overview. In: Civil Engineering Applications of Ground Penetrating Radar. s.l.:Springer 1341 Transactions in Civil and Environmental Engineering, pp. 361-371.

1342 Tracy, S. R., Black, C. R., Roberts, J. A. \& Mooney, S. J., 2011. Soil compaction: a review 1343 of past and present techniques for investigating effects on root growth. Journal of the 1344 Science of Food and Agriculture, Volume 91, pp. 1528-1537.

1345 Tracy, S. R. et al., 2010. The X-factor: visualizing undisturbed root architecture in soils 1346 using X-ray computed tomography. Journal of Experimental Botany, 61(2), pp. 311-313.

1347 Trowbridge, P. J. \& Bassuk, N. L., 2004. Trees in the Urban Landscape: Site Assessment, 1348 Design, and Installation. Hoboken, NJ: Wiley.

1349 Tubbs, C. H., 1977. Root-crown relations of young sugar maple and yellow birch, s.I.: s.n.

1350 Wagar, J. A. \& Barker, P. A., 1983. Tree root damage to sidewalks and curbs. Journal of 1351 Arboriculture, Volume 9, pp. 177-181.

1352 Wagar, J. A. \& Franklin, A. L., 1994. Sidewalk effects on soil moisture and temperature. 1353 Journal of Arboriculture, Volume 20, pp. 237-238.

1354 Wang, X., Allison, R. B., Wang, L. \& Ross, R. J., 2007. Acoustic tomography for decay 1355 detection in red oak trees, Madison, WI: United States Department of Agriculture. 
1356 Wang, Z. et al., 2006. Fine root architecture, morphology, and biomass of different 1357 branch orders of two Chinese temperate tree species. Plant and Soil, pp. 155-171.

1358 Weaver, J. E. \& Voigt, J., 1950. Monolith Method of Root-Sampling in Studies on 1359 Succession and Degeneration. Agronomy \& Horticulture - Faculty Publications.

1360 Wielopolski, L., Hendrey, G., Daniels, J. \& McGuigan, M., 2000. Imaging Tree Root 1361 Systems In Situ. s.I., s.n., pp. 642-646.

1362 Wildenschild, D. et al., 2002. Using X-ray computed tomography in hydrology: systems, 1363 resolutions, and limitations. Journal of Hydrology, Volume 267, pp. 285-297.

1364 Williams, R. E., Shaw, C. G., Wargo, P. M. \& Sites, W. H., 1986. Armillaria root disease. 1365 s.I.:US Department of Agriculture, Forest Service.

1366 Wilson, B. F., 1964. Structure and growth of woody roots of Acer rubrum L.. s.I.:Harvard 1367 University, Harvard Forest.

1368 Zenone, T. et al., 2008. Preliminary use of ground-penetrating radar and electrical 1369 resistivity tomography to study tree roots in pine forests and poplar plantations. 1370 Functional Plant Biology, Volume 35, pp. 1047-1058. 